\title{
Does Institutional Quality Matter for Multidimensional Well-Being Inequalities? Insights from Italy
}

\author{
Antonella Rita Ferrara ${ }^{1}$ (D) $\cdot$ Rosanna Nisticò $^{2}$ (D)
}

Accepted: 10 April 2019 / Published online: 23 April 2019

(c) The Author(s) 2019

\begin{abstract}
Our paper aims to shed light on regional multidimensional well-being inequalities in Italy. We first decompose the Theil index in its "within" and "between" components and we find that disparities in multidimensional well-being go beyond the historical GDP divide between the Centre-North and the South of Italy: "within" multidimensional well-being inequalities result to be as relevant as inequalities "between" these sub-national areas, suggesting that territorial-specific factors may be at work. Then, using a regional panel in the period 2004-2012, we analyse the relationship between multidimensional well-being disparities and regional institutional quality in terms of voice and accountability, government effectiveness, regulatory quality, rule of law, and corruption. We find that institutional quality matter in affecting regional multidimensional well-being inequalities and the effect varies heterogeneously accordingly to the level of public expenditure, institutional dimensions, and spatial spillovers. These findings indicate local policies could be better targeted to reduce gaps and increase expenditure efficiency, foremost among which are anti-corruption actions and measures to enhance the effectiveness of regulatory interventions, especially in regions which are lagging behind.
\end{abstract}

Keywords Institutional quality $\cdot$ Multidimensional well-being inequalities $\cdot$ Spatial correlation

\section{Introduction}

During the last few decades, the social sciences have increasingly shared the idea that "institutions matter" for studying the development patterns of different countries (Aoki 2001). A debate in both academic and public policies arenas has grown in parallel, focussing on economic and social progress, and examining the "multidimensional

Antonella Rita Ferrara

antonella.ferrara@ec.europa.eu

Rosanna Nisticò

rosanna.nistico@unical.it

1 European Commission, Joint Research Centre (JRC), Ispra, Italy

2 Department of Economics, Statistics and Finance, University of Calabria, Ponte Pietro Bucci, Cubo 1/C, Rende, Italy 
nature of well-being", which involves the non-economic dimensions of people's quality of life, other than their economic resources. This broader concept of development has gained ground compared to that based solely on GDP growth (Stiglitz et al. 2009; Nardo et al. 2008).

The standard theory of perfect competition under complete contracts as well as the longestablished traditional factor endowments (such as physical capital, human resources, technology transfer, investments in infrastructures), appear unable to accommodate a number of important economic phenomena. ${ }^{1}$ In particular, the observation and analysis of the limited returns of the traditional development strategies in reducing GDP disparities between countries and regions have focused attention on the importance of the role played by the institutional context in which policies are implemented.

Results in empirical research across countries provide evidence that institutions shape economic growth and development (Hall and Jones 1999; Acemoglu et al. 2001, 2005; Vijayaraghavan and Ward 2001; Rodrik et al. 2004). ${ }^{2}$ These studies assimilate institutions into formal rules, incentives and constraints, the rule of law, the protection of private property, and the safeguard of human rights, and government policies. Formal institutions are flanked by informal institutions (norms, values, and conventions), considered to be essential factors for generating trust and cooperation between agents: together they determine the institutional environment in which agents operate (North 1990; Amin and Thrift 1995; Aoki 2001; Rodríguez-Pose and Storper 2006). The density or thickness of informal local institutions is considered to be a factor influencing the implementation of local and regional development policies as it affects the "learning" capacity of the agent and ability to take advantage of economic and social externalities, thus facilitating agglomeration and clustering in each territory (Amin and Thrift 1995; Storper 1997; Rodríguez-Pose 2013). The presence of virtuous informal institutions is identified by many scholars of local development as an essential ingredient in the formation of industrial districts in Central and Northern Italy (Trigilia 1990; Dei Ottati 1994).

Our hypothesis is that, by defining incentives and constraints on human behaviour as well as by reducing uncertainty and information costs, institutions not only influence the conditions for the development of economic activity but also improve multidimensional well-being domains. Following this approach an enhancement of environmental quality might be achieved by an efficient enforcing system which limits pollution, promotes energy consumption from renewable sources, and incentivises separate waste collection, leading to an improvement in the quality of life of people in addition to benefitting food production and thereby, global food security. Along the same lines, a less corrupted environment might encourage firms to invest more in economic activities and might foster investment in education unless people perceive that returns from education depend on their own skills and merits. A system of stricter health rules and policies that foster disease prevention, that deters drug consumption, and promote healthy nutritional behaviours might have positive effects on health and well-being, and in turn, savings in public health costs by reducing illness. According to the Seventh report on economic, social, and territorial cohesion (European Commission 2017, p. 136), "the quality of government strongly influences people's health, their access to basic services, social trust, and political legitimacy. It helps

\footnotetext{
1 See Solow (1956) for the neoclassical growth approach, Romer (1986) and Lucas (1988) for the endogenous growth theory. For comprehensive reviews see Barro (1997); Aghion and Durlauf (2005).

${ }^{2}$ For a survey of this literature see Dellepiane-Avellaneda (2010).
} 
to explain why living conditions vary between countries and regions with much the same level of GDP per head".

Furthermore, economists agree that institutions should become part of regional development strategies in order to improve the effectiveness of the latter in reducing regional economic inequalities: in Europe, public interventions that have overlooked the institutional dimension have led to sharper disparities in GDP growth between regions since the early 1990s (Rodríguez-Pose 2013; Rodríguez-Pose and Tselios 2015; Iammarino et al. 2018). In addition, the European Commission has remarked upon the importance of assessing the effectiveness of policies aimed at reducing regional disparities and increasing homogenously not only in terms of GDP growth, but also of the well-being of people. Indeed, European regional policy funds are not limited to having an impact on the economic dimension of European regions, but they also contribute to the broader aims of the Europe 2020 strategy for smart and inclusive growth in spheres such as environmental quality, sustainability, and combating poverty and social exclusion (European Commission 2014).

All in all, these arguments highlight two main points: (1) the need to go beyond GDP in measuring economic and social progress, and (2) the importance of the role played by the institutions in reducing regional economic inequalities. In this context, there is room for new research aimed at analysing the shapes and nature of multidimensional well-being disparities, along with disparities in GDP, and disentangling how they are affected by the institutional quality. This allows to figure out what happens when both economic factors, such as income and production, and non-economic factors (e.g.: health, environment, education, personal security, and others) are jointly considered.

The aim of this article is to contribute to this strand of research by taking Italian regions as a case study. The situation of Italy is particularly interesting in the debate on regional disparities in Europe because of the wide and persistent sub-national differences in terms of both production and quality of life dimensions (Cannari and Franco 2011; Iuzzolino et al. 2011; Brandolini and Vecchi 2011). From a strictly economic point of view, many indicators geographically group Italian regions into more developed areas clustered in the Centre-North of the country, while economic backwardness is still common in the South of Italy, which is known as the Mezzogiorno. ${ }^{3}$ One-third of the national population lives in the Mezzogiorno which produces just 22\% of national GDP. Per capita GDP level is 18.2 thousand euros in Southern regions, $44.2 \%$ lower than in the Centre-North, whilst labour productivity and the employment rate are, respectively, 19\% and 30\% lower than the Centre-North. Mezzogiorno regions contain two-thirds of the poor in Italy and 53\% of the national youth unemployment. Beyond the (economic) dualism between the Centre-North and the South of the country, Italian regions exhibit wide differences in non-economic domains of well-being, with underutilisation of resources in the lagging regions. Campania and Lombardy have similar population densities but they are eleven and seven times higher than the Valle d'Aosta (38.7 inhabitants per square kilometre) and Basilicata (56.3), respectively. ${ }^{4}$ The percentage of students aged 15 who reached levels of performance higher than "level 2", defined by the 2015 OECD-PISA survey as "the baseline level of science proficiency that is required to engage in science-related issues as a critical and informed

\footnotetext{
${ }^{3}$ Sub-national areas in Italy include eight regions (Valle d'Aosta, Piedmont, Lombardy, Trentino-Alto Adige, Friuli-Venezia Giulia, Liguria, Emilia Romagna and Veneto) for the North; four regions for the Centre (Toscana, Marche, Umbria and Lazio) and eight regions for the South, or Mezzogiorno (Abruzzo, Molise, Campania, Puglia, Basilicata, Calabria, Sicily, and Sardinia).

${ }^{4}$ Source: www.tuttitalia.it.
} 
citizen" (OECD 2016, p. 72), is about $33 \%$ in Campania compared to $59 \%$ in Lombardy. ${ }^{5}$ The highest percentages of children up to the age of 3 using child-care services are found in Emilia Romagna (26\%) and in Valle d'Aosta (28\%) while the lowest percentage is that of Calabria $(2.1 \%)^{6} ; 60 \%$ of people aged 14 and over have a sedentary lifestyle in Sicily and Campania as opposed to $14 \%$ in Trentino-Alto Adige. ${ }^{7}$ Moreover, the percentage of acute care hospital discharges of patients who have required hospitalization in a region different from that of residence, which is considered an index of people distrusting their regional health system, is $3 \%$ in Lombardy compared to $20.5 \%$ in Basilicata and Calabria, and 22\% in Molise. ${ }^{8}$

It comes out that citizens subject to the same institutional and legal order, who are expected to enjoy the same levels and quality of essential public services, such as a comparable quality of education, health services and justice efficiency, are actually exposed to different livability conditions depending on the region where they live. Indeed, measuring well-being inequalities is at least as relevant for policy as economic performance and income disparities because it entails principles of equity and citizenship as well as happiness and life satisfaction.

However, a large amount of public expenditure is spent by sub-national authorities in many fields of intervention linked to quality of life (Fig. 1). This particularly occurs in environment protection and health where the percentage of regional government expenditure out of the total general government expenditure reaches, respectively, $82 \%$ and $80 \%$. This percentage is more than three times higher for health in Italy than the EU-27 average. A not negligible share of the total national government expenditure is spent by Italian regional governments on the functions of "housing and community amenities" and "economic affairs, including transport", respectively about $60 \%$ and $40 \%$. In the other fields of intervention, the share of local public expenditure in Italy is below the European average: "recreation, culture and religion", "education", "general public services", "public order and safety", and "social protection". This evidence suggests that examining the local implementation of national policies in the fields of intervention affecting multidimensional well-being is worth of interest.

This paper strives to assess whether there is a relationship between regional disparities in terms of multidimensional well-being and regional institutional quality. Moreover, the paper investigates how various levels of institutional quality combined with different regional expenditure endowments are correlated with regional differences in well-being. To measure multidimensional well-being at the regional level, the analysis takes advantage of an existing synthetic indicator (RWBI) constructed by combining ten dimensions of quality of life. Previous studies of Italian sub-national disparities in well-being in the literature mainly focus on indices only capturing the economic dimension (Berloffa and Modena 2012) or a lower number of well-being dimensions (Brandolini and Vecchi 2011; Iuzzolino et al. 2011; Ferrara and Nisticò 2013). Institutional quality is also measured by a synthetic index (IQI) defined by Nifo and Vecchione (2014), available at Italian regional level, which combines five domains (voice and accountability, government effectiveness, regulatory quality, rule of law, and control of corruption), so the economic dimension of institutional quality can be taken into consideration.

\footnotetext{
${ }^{5}$ OECD (2016), Vol. I, Annex B2.

${ }^{6}$ Source: Istat-DPS data base, www.istat.it/it/archivio/16777.

${ }^{7}$ Source: Dati.istat.it.

${ }^{8}$ Source: Istat-DPS data base, www.istat.it/it/archivio/16777.
} 
The analysis is structured in three steps. Firstly, we provide evidence on multidimensional well-being inequalities "within" and "between" Italian subnational areas. Unlike Ferrara and Nisticò (2015) who analysed the evolution of regional multidimensional wellbeing dispersion relying upon RWBI index, we use the Theil index in order to decompose well-being inequalities in "within" and "between" subnational areas components. Secondly, differing from other studies, we explore whether the institutional quality and regional public expenditure affect regional multidimensional well-being inequalities. Thirdly, the concept of spatial autocorrelation is introduced and explored in the estimated models.

The article is organized as follows. The second section presents a brief survey of the recent literature with a focus on the concept of institutions and their effects on well-being, the definition of well-being indicators, and the state of play in quality of life measurement in Italy. Then data and methods are described in the third section. After this, the fourth section focusses on the Theil's index decomposition and the fifth section introduces the spatial dimension. The sixth discusses the role of institutional quality in explaining regional wellbeing inequalities, and lastly, the seventh section presents the conclusions.

\section{Related Literature}

The multidimensional nature of both well-being and institutional quality means that this paper relates to several strands in the literature, so the main contributions are reviewed along four lines of interpretation. First, the evolution of the concept of institutions is examined. Then, the main studies assessing the link between institutions and well-being are outlined. Finally, the state of the art on well-being in Italy for both the definition of composite measures and the empirical applications are presented.

\subsection{Alternative Views of Institutions}

In addition to stating that "institutions matter", social scientists have increasingly taken up the task of conceptualizing institutions. Although a comprehensive review of the different approaches to institutions in social sciences is beyond the aims of this paper, it is worth noting that the current literature has not agreed on a common definition. However, scholars in economics share the idea that they influence transaction costs and therefore efficiency.

There are at least three different views of institutions in economics (Aoki 2001). One definition is by North (1990), who identifies institutions as the "rules of the game" agents conceive in order to discipline their interactions, that is, both formal rules (such as constitution, regulations, legal system, political rules, formal contracts), and informal norms (i.e. norms, moral codes, conventions). They define both allowed and not allowed actions, thus providing a guide for human behaviour and thereby reducing uncertainty. The enforcement system and the sanctioning mechanism are essential for the proper functioning of the institutions. In North's view, there is a clear distinction between institutions (rules and norms) and organizations (groups of people united by a common purpose of achieving a goal). The focus on rules is shared by Hodgson's view (2007, p. 331) of institutions as 'enduring systems of socially ingrained rules' and, beyond a strictly economic definition, by Ostrom (Ahn and Ostrom 2009; Ostrom 2015). She defines institutions as the prescriptions humans use in repetitive situations (within families, neighbourhoods, markets, firms, sports leagues, churches, private associations, and governments) organized by rules (must, must not, or may), norms (prescriptions enforced by the participants themselves through 


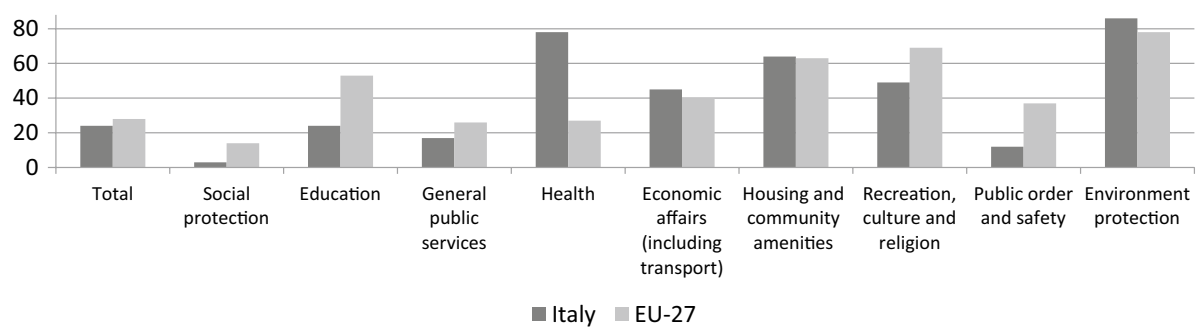

Fig. 1 Subnational government expenditure by function (\% of total general government expenditure), 2013. Source: Our elaboration on Eurostat data

social sanctions) and strategies (plans that individuals make within the existing structure of incentives). From this perspective, the role of institutions in shaping economic development is pervasive: "Institutions provide the incentive structure of an economy; as that structure evolves, it shapes the direction of economic change towards growth, stagnation, or decline" (North 1991, p. 97).

The issue of enforcement is crucial for an alternative definition which sees institutions as a point of equilibrium in a repeated game (Greif 1989, 1998; Greif et al. 1994; Hurwicz 1996; Aoki 2001): when institutions become established, they represent a self-enforcing equilibrium, which is not imposed by an exogenous mechanism, but is the result of a game where each agent has no incentive to deviate from her strategy as long as all the other agents are expected not to change the prescribed strategy.

The common reference to institutions as organizational establishments is mirrored in a third definition. This not only includes the rules in force in a given society or economic system, but also agents such as "industry associations, technical societies, universities, courts, government agencies, legislatures, etc." (Nelson 1994, p. 57), whose role is to apply the rules and ensure they are respected by others, as well as the organizations and the means they use to perform this task. Unlike North's view, there is no distinction in this interpretation between the rules and the players of the game. A similar approach characterizes both the "historical" and "rational choice" institutionalism schools of thought in political science. The former associates institutions with organizations and the web of rules, formal and informal procedures, routines, and norms and conventions that are promulgated by formal organizations; the latter stresses "the importance of property rights, rent-seeking, and transaction costs to the operation and development of institutions" (Hall and Taylor 1996, p. 943). It is heavily influenced by the transaction cost economics theory of Williamson (1989) according to which the comparative analysis of both transaction and production costs between alternative forms of governance, firm versus market, determines the agent's choice between "make" or "buy".

In the sociological literature dealing with the relationship between institutions and development, some scholars distinguish between "culturalist" and "institutionalist" approaches (in a strict sense) (Trigilia 2015). The institutionalist, which includes contributions from North (1990), Putnam (1993), Landes (2000), Harrison and Huntington (2000), emphasises shared values and beliefs in a given society (commitment, honest behaviour in business, civicness, people's propensity to cooperation). According to North, institutions arise out of the shared values of society and change following a slow, path-dependent process of adjustment of values. The well-known Putnam study (1993) refers to the Italian regions and states that formal institutions work better in the Centre-North than in South of the country, due to the existence in the former of greater social capital (civicness and 
propensity to cooperate). The reasons for the different endowment of social capital in the two Italian subnational areas are to be found in their differing long-term history. ${ }^{9}$ On the contrary, according to the "institutionalist" point of view, development depends on the quality of government, determined by institutions that guarantee the enforcement of contracts, safeguard the protection of property rights, and ensure the production of collective goods. Such institutions, defined as "inclusive" by Acemoglu et al. (2005), do not arise from cultural values but depend on the ability of inclusive political regimes to limit the tendency of the élites to shape the economic rules in favour of their interests, and in contrast, are able to encourage the active participation of members of the society and to promote citizenship rights.

According to Aoki (2001, p. 10), "which definition of an institution to adopt is not an issue of right or wrong; it depends on the purpose of the analysis". As a matter of fact, studies dealing with the relationship between institutions and economic development refer to institutions in different ways: to give a few examples, they are "social infrastructures", i.e. rules and government policies that determine the economic environment in which economic agents operate (Hall and Jones 1999); the incentives of and the constraints on economic actors (Acemoglu et al. 2001, 2005); a number of measures of institutional infrastructure such as the security of property rights, governance, political freedom, and government consumption (Vijayaraghavan and Ward 2001); the rules of the game in a society (property rights and the rule of law) and their conduciveness which lead to desirable economic behaviour (Rodrik et al. 2004).

This paper considers the IQI index (Nifo and Vecchione 2014) as a measure of institutional quality. IQI is inspired by the World Governance Indicator (Kaufmann et al. 2011) which is a composite indicator designed to measure the quality of governance.

\subsection{Institutions and Well-Being}

The role of institutional factors in determining spatial inequality in multidimensional wellbeing, measured by jointly considering income and non-income aspects of people's quality of life, has hardly been investigated so far. Empirical contributions have mainly provided evidence on the link between institutional factors and individual areas of well-being. However, most of these studies have been carried out at national level but research at the subnational scale has been increasing in recent years.

Cross-country studies emphasise the fact that nations with more efficient norms and impartial and non-corrupt governments exhibit a higher level in some well-being dimensions (Holmberg et al. 2009; Mauro 1995; Mo 2001; Morse 2006; Welsch 2004; Mauro 1998; Gupta et al. 1998; Helliwell and Huang 2008; Ott 2010).

Holmberg et al. (2009) find a positive correlation between an index of quality of government which takes into account three widely used variables (government effectiveness, rule of law, and corruption perception), and 18 out of 22 different variables at country level measuring important social outcomes in five areas (health, environmental, economic and societal outcomes, and subjective feel goodness) each of which considered in a separate regression. Mauro (1995) investigates the effect of corruption on the ratio of investment to

\footnotetext{
9 As Ostrom (2008, p. 74) observes, "For some scholars, such as Putnam and his colleagues (1993), social capital was the independent variable they identify as affecting the dependent variable of institutions." (Emphasis as in the original).
} 
GDP for 58 countries and finds a negative relationship. The impact of corruption on both GDP and two well-being dimensions is also analysed by Mo (2001), who carries out an empirical analysis on 46 countries. The results show that a $1 \%$ increase in the corruption level reduces the growth rate by about $0.72 \%$. He finds that the most important channel through which corruption affects economic growth is political instability, which accounts for about $53 \%$ of the total effect; it reduces the level of human capital (about $9.7 \%$ of the rate of productivity reduction is caused by the human capital channel) and the share of private investment on GDP (about $28 \%$ of the growth rate reduction is due to the investment channel). In presence of corruption, people are incentivized to allocate their talent and effort in rent-seeking activities rather than in productive investments which include accumulating capital, knowledge, and skills. Furthermore, the author highlights how corruption, by favouring particular social classes, stimulates inequality in opportunities, thereby generating psychological frustration in the underprivileged.

Some cross-national analyses explore the link between (bad) institutional quality and environment. Morse (2006) provides evidence of the detrimental effect of corruption on various aspects of environmental sustainability (environmental governance, private sector responsiveness, participation in international collaborative efforts). Furthermore, Welsch (2004) estimates the effect of corruption on pollution, finding a monotonically increasing relationship between the two phenomena, particularly strong at low-income levels. He suggests that developing countries should reduce corruption in order to improve both their economic and environmental performances. Cross-country studies have found that low institutional quality adversely affects the composition of government spending, particularly by reducing government spending on education and health (Mauro 1998) and by increasing inequality and poverty (Gupta et al. 1998). The quality of government also affects subjective well-being: Helliwell and Huang (2008) find that the effect of good government is the most important variable explaining international differences in life satisfaction; while a comparison of 127 nations (Ott 2010) shows that the quality of governance has strong correlations with the average happiness of citizens and it appears to be more important for happiness than the size of government.

The relationship between government quality and single dimensions of well-being at the regional level has been the subject of recent research. Cortinovis et al. (2017) demonstrate that good quality of both formal institutions and social capital (trust and active participation in bridging-type groups) increase the probability of European regions diversifying economic activity into new sectors. The quality of government is found to play a major role in determining the returns of European investments and its relevance increases with the level of cohesion expenditure (Rodríguez-Pose and Garcilazo 2015). In a similar vein, Crescenzi et al. (2016) explore the link between regional quality of government and the returns of different types of infrastructure investments for European regions whilst Rodríguez-Pose and Di Cataldo (2015) assess how institutions shape the capacity of regions to innovate. Other research also confirms the positive correlation between institutional quality and subjective well-being at the sub-national scale (Frey and Stutzer 2000; Rodríguez-Pose and Maslauskaite 2012).

Various studies have examined the relationship between institutions and income spatial inequality, defined as "income inequality across geographical or administrative units within a territorial entity (e.g., country, region)" by Ezcurra and Rodriguez-Pose (2014, p. 1732). Empirical evidence shows that there is a negative relationship between institutional quality and regional income disparities (Kyriacou and Roca-Sagalés 2013; Ezcurra and Rodrìguez-Pose 2014). Kyriacou and Roca-Sagalés (2013) analyse a sample of 22 OECD countries over a period of 10 years using different indicators of government quality as well 
as different measures of spatial income disparities over the period 1996-2006. The study by Ezcurra and Rodrìguez-Pose (2014) considers forty-six countries with different levels of economic development over a period of 11 years. Both of the abovementioned studies support the hypothesis that good government quality contributes to lower regional disparities.

Institutional quality also influences the effect of fiscal decentralization on regional disparities as shown in an empirical study based on 24 OECD countries over the period 1984-2006 (Kyriacou et al. 2015): regional convergence in countries with high-quality institutions is promoted by fiscal decentralization while the opposite effect occurs in countries with low-quality institutions experiencing wider regional disparities in the presence of fiscal decentralization.

With regard to Italy, several studies analyse the relationship between institutions and sub-national disparities looking at the economic and human development since the country's unification in 1860 (Iuzzolino et al. 2011; Brandolini and Vecchi 2011; Felice and Vasta 2015; Felice 2017). These studies emphasise that for better or worse institutions have been crucial in determining the size of the economic and social imbalance between the Mezzogiorno (South) and the Centre-North sub-national areas.

This non-exhaustive review of the literature highlights the point that institutional quality is related to economic progress, quality of life, and spatial income inequalities. The link between institutional quality and regional multidimensional well-being disparities is related to how regions design and implement public policies in the field of interventions associated to well-being dimensions, especially in those fields where the share of subnational government expenditure out of general government expenditure is high.

\subsection{Measuring Multidimensional Well-Being}

Despite a big effort being made during the last few decades, a unique and universally accepted cross-country measure in the data collection and definition of multidimensional well-being indicators is still lacking. This issue is even more marked when considering the sub-national level and longer time spans in diachronic analysis of well-being disparities.

Multidimensional well-being measurement is heavily based on the composite indicators approach which combines elementary variables into synthetic indices adopted to represent each well-being dimension that might then be aggregated into an overall synthetic indicator. This approach has advantages and disadvantages (Saisana et al. 2005; Hagerty and Land 2007; Nardo et al. 2008; Dialga 2017). On the one hand, composite indicators favour a synthetic analysis of the phenomenon under study as they can be interpreted more easily than a large battery of variables, although preserving the original information. Moreover, they allow deeper examination of their intermediate components and facilitate both communication and accountability. On the other hand, a number of methodological and theoretical issues arise. First of all, indicator reliability in representing the underlying phenomenon is strictly related to the accuracy of its definition: a poorly defined synthetic measure may lead to biased results and wrong policy decisions. Secondly, the choice of variables and weights may imply a certain degree of subjectivity by the researcher.

A standard well-known measure of human well-being available for cross-country studies is the Human Development Index (HDI), which has been calculated by the UNDP since 1993. This index provides comparable cross-country data for a long time interval which makes it highly suitable for assessing convergence and inequalities in quality of life. Yet, when it is used for comparative studies regarding more industrialized countries, it is often 
"augmented" to take into account a wider and more appropriate spectrum of quality of life dimensions (Marchante and Ortega 2006; Stiglitz et al. 2009).

The need for a measure of multidimensional well-being is currently high on the agenda of many international institutions. Among the most interesting initiatives, the Better Life Index by the OECD provides a set of indicators grouped into eleven dimensions of wellbeing and an interactive tool that allows users to visualize data of more developed and some emerging economies, at national and regional level, and to obtain a synthetic measure of multidimensional well-being as an arithmetic mean. Users are also allowed to set the weights of the different dimensions according to the importance assigned to each aspect of the quality of life. In addition, the European Commission has promoted a number of "Beyond GDP" initiatives aimed at developing indicators and improving the analysis of economic and social progress, including environmental sustainability. The European Statistical System Committee (ESSC) has established a "sponsorship group on measuring progress, well-being, and sustainable development" and has developed a set of quality of life indicators for EU countries covering ten well-being dimensions. Many countries have carried out programmes for developing comprehensive statistics to measure multidimensional well-being. ${ }^{10}$ Among the best-known initiatives undertaken by national governments, it is worth mentioning the French government decision in 2009 to commission a group of experts, including Nobel laureates, to report on the measurement of economic performance and social progress that became a milestone in the literature on the subject. The Commission, chaired by Joseph Stiglitz, Amarthya Sen, and Jean Paul Fitoussi, does not provide a synthetic well-being indicator but firmly claims that well-being is a multidimensional concept and recommends integrating the measurement of economic aspects with those related to sustainability, social cohesion, and non-income aspects of life.

\subsection{Multidimensional Well-Being Inequalities Across Italian Regions}

In Italy, the Bes (i.e. the Italian acronym for "fair and sustainable well-being") project which has been carried out by the National Institute of Statistics (Istat) and the National Council for Economy and Labour (Cnel) since 2010, has been constructing a database which considers twelve dimensions of quality of life. The Bes project does not attempt the final step of providing a synthetic measure of multidimensional well-being.

A pioneering contribution to the measurement of multidimensional well-being in Italy is QUARS (2012), a composite indicator proposed by the "Sbilanciamoci!" campaign, which combines seven dimensions of regional well-being as an arithmetic mean (Environment, Economy and Employment, Health, Rights and Citizenship, Education and Culture, Gender Equality, and Participation). This campaign started in 2003 to measure the quality of life and the economic development of Italian regions at yearly intervals by using a set of indicators regarding several crucial dimensions of economic and social progress such as environment, work conditions, equal opportunities, civic participation, health, and culture, and a report was periodically published until 2012.

A number of other composite indicators have been constructed in studies approaching multidimensional well-being disparities and their dynamics in Italian regions. The "real freedom index" (Capriati 2011) combines six variables in 3-year intervals from 1998 to

\footnotetext{
10 As regards European countries, see the interactive map at http://ec.europa.eu/environment/beyond_gdp/ news_map_en.html.
} 
2007, computed as the arithmetic mean of the standardized variables, and assesses convergence patterns across Italian regions through the coefficient of variation of the composite indices. Ferrara and Nisticò (2013) construct a "well-being index" at regional level over the period 1998-2008, taking into account six different dimensions of quality of life (life and health expectancies, knowledge and education, material conditions, equal opportunities in the labour market, competitiveness, quality of socio-institutional context). A composite indicator is calculated for each dimension as a weighted mean of the original variables in order to obtain two synthetic indices of multidimensional well-being: the "augmented human development index", similar to Marchante et al. (2006), and the overall "well-being index". Adopting the same methodology at the Nuts 3 level, Di Berardino et al. (2016) calculate both a "human development index" and a "well-being index" and they also explore the dynamics of multidimensional well-being disparities among Italian provinces over the period 2000-2007 and the role of industrial districts by carrying out a $\beta$-convergence analysis. The "regional well-being indicator" proposed by Ferrara and Nisticò (2015) for Italian regions synthesises 10 different dimensions (culture and free time, education, employment, environment, essential public services, health, material living conditions, personal security, research and innovation, social relations). A synthetic indicator for each dimension of wellbeing is calculated for each region every year from 2004 to 2010, and then an overall composite index of well-being is generated by applying a two-step principal component analysis. Other papers examine regional disparities by resorting to an extended version of the HDI along with GDP and a number of other economic and social indicators (Felice 2007; Brandolini and Vecchi 2011; Iuzzolino et al. 2011) by adopting a historical perspective.

The entity and the persistence of subnational spatial inequalities make the Italian situation an interesting case-study in the international framework. The size of disparities between leading and lagging regions in both economic and other dimensions of well-being is indeed among the highest in Europe. This is mirrored in different availability and quality of several essential public services accessible for people living in backward regions (Cersosimo and Nisticò 2013; Ferrara and Nisticò 2013). Because of the persistence of such disparities, alternating between stability and changes since the country's unification in 1860 , a number of studies have adopted a historical perspective in dealing with spatial well-being inequalities in Italy.

The productive gap between northern and southern regions is indeed the most macroscopic and highly debated problem in Italian national history. Starting from the unification of the country, Iuzzolino et al. (2011) find that there is no evidence of a convergence process across Italian regions except for two periods: the interval between the unification and the mid-1880 and the years 1950-1972. National government policy has played a central role in exacerbating or attenuating the divide between the two sub-national areas. In the South of Italy, the end of special investment programmes in 1992 and the increase of labour costs, until then lower than in the rest of Italy owing to the existence of "wage cages", have strongly hit the competitiveness of Mezzogiorno regions in recent decades. Moreover, a long period of stagnation, with a restrictive fiscal policy, has had an especially marked impact on the economy of the South, which was more dependent on public resources than the rest of the country. Consequently, the Mezzogiorno has fallen further behind in terms of per-capita GDP over the last 20 years, giving rise to a slowdown in the convergence process.

According to Felice (2017), a different quality of institutions which is more "inclusive" in the Centre-North than in the South, as stated by Robinson and Acemoglu (2012), has facilitated the economic and social progress in the former area. Felice (2007) examines regional well-being disparities by considering seven social indicators, including the UN 
Human Development Index (HDI) and an "improved" Human Development Index, over a period of 130 years (1871-2001). It emerges that the regional convergence process was interrupted during the 1980s and 1990s.

Felice and Vasta (2015) extend the analysis in order to consider the new HDI introduced by the UN in 2010 . The interpretative hypothesis explaining the evolution of regional imbalances relies upon the distinction between "active" and "passive" modernisation (Cafagna 1988), where the former occurs "when one or more political or social actors take up the challenge and engage in modernizing the country" (p. 45) while the latter takes place when "a society embarks upon some sort of modernization ... without implementing a proper strategy, but rather as a result of an adaptive-sub-optimal pattern" (ibidem). According to the authors, Southern Italy improved in social indicators of quality of life thanks to passive modernisation, which led to greater convergence with Northern levels of life expectancy, yet it was less successful in education and even ineffective in terms of GDP. Brandolini and Vecchi (2011) show that economic growth was accompanied by a long-term reduction in inequality, though reversed over the last two decades, whilst the increase in life expectancy was remarkable, placing Italians first in the international ranking. Educational achievements improved considerably as well although no more impressively than in other countries.

A number of studies on multidimensional spatial well-being inequalities in Italy have been carried out by means of different composite indicators with the aim of providing a ranking of Italian regions in terms of well-being or assessing convergence over time (Di Berardino et al. 2016; Ferrara and Nisticò 2013, 2015). During the 10 years before the advent of the economic and financial crisis, Italian regions grew more similar to each other as a consequence of a convergence process both in terms of GDP and multidimensional well-being, albeit large disparities still persist across regions at the end of the period (Ferrara and Nisticò 2013). The reduction of disparities is also confirmed at the provincial level (Di Berardino et al. 2016). The convergence trend shows a slower narrowing of the productive gap by southern regions than for multidimensional well-being. However, in some aspects of quality of life, such as the quality of the socio-institutional context, age and gender discrimination in the labour market, and the degree of competitiveness, the results of the analysis highlight the need for more incisive policies in order to foster the convergence process. Regarding the ranking of Italian regions, differences in multidimensional wellbeing do not necessarily overlap those based on per capita GDP even though the historical Italian divide, with the southern regions occupying the bottom positions, reappears for most of the well-being dimensions. It emerges that in addition to the economic dualism, equally relevant gaps exist in a number of other aspects of well-being which depict a more realistic situation in the country than that stemming from using only the productive dimension (Di Berardino et al. 2016; Ferrara and Nisticò 2015).

\section{Well-Being, Inequality, and Institutional Quality in a Multidimensional Perspective: Data and Methods}

The empirical analysis could be broadly split into three parts. The first is a descriptive part, where the additively decomposable property of the Theil index is exploited to investigate inequality within and between Italian regions. A second explanatory part analyses the relationship between regional well-being disparities and institutional quality in a panel setting. Moreover, the paper disentangles the effect by each institutional quality dimension and assesses whether there is a heterogeneous influence due to the level of regional per 
capita expenditure in well-being dimensions. Lastly, a third part adds a spatial autocorrelation component to the previous models.

We use a standard measure of inequality applied to the above-described multidimensional regional well-being index (the RWBI), calculated for the Italian regions by Ferrara and Nisticò (2015) over the period 2004-2010. The indicator considered here refers to an updated version which covers two additional years (up to 2012) compared to the original RWBI data. A synthetic index is also adopted to represent the quality of government, that is, the Institutional Quality Index (IQI) defined by Nifo and Vecchione (2014).

The choice of resorting to the RWBI is made for a number of reasons. Firstly, RWBI considers the widest spectrum of domains and variables (57 original variables) that have until now been used for the construction of a synthetic well-being index for Italian regions (see Figs. 6 and 7 in "Appendix A" for a complete list of the variables considered). Secondly, it is computed for a long-enough period covering 9 years (2004-2012). Thirdly, the problem of subjectivity in choosing the variables to be included in the synthetic index is mitigated by the fact that the same dimensions are also contemplated in the Istat BES project. Compared with the BES domains, only those dimensions for which variables are not available at the regional level for the entire period of interest are excluded from the analysis, i.e.: "subjective well-being", "politics and institutions", and "landscape and cultural heritage" whereas the RWBI also includes the "culture and free time" dimension. ${ }^{11}$ Moreover, the methodology used for constructing the RWBI avoids subjective weighting schemes, which are a frequent weakness in building composite indicators. At the same time, it evaluates the internal consistency of both the indicators of overall well-being and the variables in the intermediate indices. In fact, the RWBI is obtained by means of a twostep principal component analysis: in the first step, the original variables, grouped into 10 sets each describing a well-being domain, are reduced to synthetic indicators by the PCA (see Fig. 7 in "Appendix A"); in the second step, these sub-indices are used as new variables to calculate the overall well-being indicator applying the same method. Therefore, the aggregation of the original elementary indicators is data-based and the resulting summary indicators account for a large part of their variance (Nicoletti et al. 2000).

As with multidimensional well-being, the measurement of institutional quality is not easy. Different indicators are available at country level, such as the widely-used World Governance Indicator constructed by Kaufmann et al. (2011), the Institutional Quality Dataset (Kunčič 2014), and the European Quality of Government Index, proposed by Charron et al. (2014), which has also been calculated at a sub-national level in twenty-seven European Union countries for 2 years (2010 and 2013). It has been shown that withincountry regional variation in Quality of Government is at times as or more important than cross-country variation: from our point of view, it is worth noting that the difference between two Italian regions, Trentino-Alto Adige in the north and Campania in the south of Italy, is greater than the gap between Denmark and Portugal (Charron et al. 2014). Regarding Italy, the Institutional Quality Index (IQI, Nifo and Vecchione 2014), inspired by the abovementioned World Governance Indicator, is available at sub-national level for the whole period 2004-2012. It considers five dimensions measured by objective sub-indicators (see Figs. 6 and 8 in "Appendix A") ${ }^{12}$ which are: (1) Voice and accountability, which

\footnotetext{
11 For a more detailed discussion on the choice of RWBI dimensions refer to Ferrara and Nisticò (2015).

12 Data are collected from institutional sources (Istat, Interior Minister, Ministry of Economy and Finance, Ministry of Health, Ministry of Justice, Revenue Agency), research institutions (Istituto Tagliacarne, Centro Ricerche Economiche Nord Sud-CRENoS), professional registers (Il Sole 24 Ore, Legambiente, Confartigianato).
} 
synthetizes participation in public elections, the number of associations and social cooperatives, and cultural liveliness (in terms of books published and purchased in bookshops); (2) Government effectiveness, based on two indices measuring firstly the endowment of social facilities (education, healthcare and leisure) and economic infrastructures (roads, railroads, ports, airports, energy, information and communication technology, banking), and secondly the administrative capacity of local governments in designing health, waste management and environment policies; (3) Regulatory quality, which captures the effectiveness of local administrators in promoting and protecting business activity in five variables regarding the degree of openness of the economy, a composite index for the business environment (including information on entrepreneurship, the job market, tax system, market competition, bureaucracy, cooperation between firms), and four variables concerning the economic structure (business density, business starts-up/mortality, local government employees); iv) Rule of law, relying upon data on criminality (crimes reported and crimes against property), magistrate productivity, trial times, and irregular activities (degree of tax evasion and the shadow economy); v) Control of Corruption which is an inverse corruption index and it is based on three variables regarding crimes committed against the public administration, the number of local administrations overruled, and a composite index of corruption proposed by Golden and Picci (2005), respectively. All of the sub-indices are positively correlated with institutional quality.

For the purpose of this analysis conducted over a time span of 9 years, the IQI is the most suitable variable for exploring the link between multidimensional well-being inequalities and institutional quality. ${ }^{13}$

On empirical grounds, the previously described features of the Italian regional system mean the evolution of well-being disparities should be analysed by investigating whether the convergence process (Ferrara and Nisticò 2015) has brought a greater similarity between regions within each sub-national area (North, Centre, and Mezzogiorno) or between these three sub-national areas. This is why the additively decomposable property of the Theil Index is very helpful in isolating two components: inequality withinarea and inequality between-areas for GDP and the RWBI over the period 2004-2012 (methodological details on the decomposition of the Theil index are provided in "Appendix C").

In the second part of the empirical analysis, the relationship between regional differences in well-being and the institutional quality endowment of the respective region is investigated and the extent a better institutional environment may lead to higher or lower disparities in multidimensional well-being is measured. For this purpose, a measure of multidimensional regional well-being disparities is defined by computing the deviation between the Italian mean value of RWBI and the regional value. The greater this difference is, the wider the regional inequalities. Moreover, the same variable is defined as a deviation from the mean value of the RWBI in the respective subnational area in order to focus on differences at the macro area level. These two variables allow how much a region differs from the rest of the country or from the regions in the same macro area to be quantified, leading to a proxy measure of regional inequalities in well-being. The result is a strongly balanced panel dataset of twenty regions for 9 years.

\footnotetext{
13 Further details on RWBI, IQI and their regional values are provided in Appendix B. For methodological aspects, see Ferrara and Nisticò (2015) for RWBI and Nifo and Vecchione (2014) for IQI.
} 
The baseline model is represented by the following regression equation, estimated by applying a panel fixed effects model ${ }^{14}$ :

$$
Y_{i, t}=\alpha+\beta I Q I_{i, t}+\gamma t+\eta t i+\theta i+\varepsilon_{i, t}
$$

where $Y_{i, t}$ is the above-described well-being inequality measure for region $i$ at time $t$, defined either at national $(\Delta \mathrm{N}-\mathrm{Rrwbi})$ or at local subnational area level $(\Delta \mathrm{A}-\mathrm{Rrwbi})$; $\gamma \mathrm{t}$ identifies the year fixed effects, $\eta t i$ are the regional linear time trends, and $\theta i$ are the regional fixed effects. The aim is to investigate whether institutional quality affects multidimensional well-being inequalities between Italian regions. The regional and time fixed effects and the regional linear trends allow the effect of the quality of government to be isolated from any other factor related to the structural characteristics along with specific events that took place in a region at a certain point in time. All of the models are estimated with robust standard errors.

The same model is also estimated by considering each of the IQI dimensions (SubIQI) instead of the composite index: e.g., Voice and accountability, Government effectiveness, Regulatory quality, Rule of Law, and Control of Corruption (from now on Corruption).

Furthermore, assuming that the regional deviation from RWBI national or local average might not only be affected by the quality of the government but also by the "size" of government, the latter is also added to the model. To measure the regional economic endowments, the logarithm of the regional per capita expenditure at time $t$ in well-being related domains (education, environment, security, health, justice and other public services, labour market, research and innovation, culture and material conditions) is considered, with data being provided by the Italian National Institute of Statistics (ISTAT). However, since expenditure commitments might experience some synergies with the functioning of the institutional system itself, the related heterogeneity is assessed by adding the variable

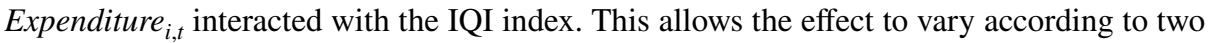
continuous variables.

For the sake of easier interpretation, when the model considers the IQI sub-indices, the expenditure is introduced as a categorical variable. The dummy variable highexp ${ }_{i, t}$ is equal to 1 if the region has registered higher expenditure in well-being related domains than the national median value for that year. Consequently, the regression equation becomes:

$$
Y_{i, t}=\alpha+\sum \beta_{1} \operatorname{SubIQI}_{i, t}+\beta_{2} \operatorname{HighExp}_{i, t}+\sum \beta_{3}\left(\operatorname{SubIQI}_{i, t} * \operatorname{HighExp}_{i, t}\right)+\gamma t+\eta t i+\theta i+\varepsilon_{i, t}
$$

In this case, the effect of each IQI component on regional multidimensional well-being disparities is given by the sum of the respective $\beta_{1}$ and $\beta_{3}$ estimated coefficients, with $\beta_{3}$ depending on the dichotomous variable $\operatorname{HighExp}_{i, t}{ }^{15}$

\footnotetext{
${ }^{14}$ The usual Hausman and Mundlak tests are performed to choose between a fixed effect rather than a random effect model. Further details on the tests are provided in the section discussing the results.

${ }^{15}$ This implies that for higher levels of expenditure $\left(\operatorname{HighExp}_{i, t}=1\right)$ the effect of each sub-index is jointly given by the respective $\beta_{1}$ and $\beta_{3}$; whereas, for lower levels of expenditure $\left(\operatorname{HighExp}_{i, t}=0\right)$, the effect is given only by the respective $\beta_{1}$. In other words, $\beta_{3}$ is the differential effect of the regions with higher expenditure levels respect to those with lower expenditure for an average level of IQI.
} 


\section{A Description of "Within" and "Between" Inequalities in Multidimensional Well-Being}

The evolution over time of the income and non-income dimensions of well-being may not necessarily be the same (Bourguignon and Morrisson 2002). This result is also confirmed by Ferrara and Nisticò (2015) finding, for the overall well-being index, that the reduction of inequalities slows down after the advent of the 2007-2008 crisis, whilst disparities in GDP have slightly increased in the aftermath of the crisis. Analogous results come from a study on European regions which finds a progressive reduction of economic disparities up until 2007 and an opposing trend after that (European Commission 2014). It is clear that results in terms of multidimensional well-being do not overlap those in terms of productive performance; the reaction to exogenous shocks seems to be rather different in the two cases, in line with studies which find a more complex interaction between well-being and economic development than a simple cause-and-effect relationship (Corsi and Guarini 2011).

The exercise of decomposition has the merit of suggesting that territorial-specific factors may be at work. Hence, when disparities are investigated in terms of GDP, the historical Italian divide between the more industrialised and economically advanced North and the backward South of Italy overshadows differences at the local level within these subnational areas so that inequalities "between" prevail over inequalities "within". On the contrary, when inequalities are measured in terms of well-being, not only has the process of convergence between the subnational areas (Centre-North and South) not been restrained, but the inequalities "within" are also as relevant as inequalities "between" areas. This is a crucial point as well-being is inherent to quality of life and this is very uneven in different regions within the same country even though there is political, fiscal, and administrative homogeneity. In other words, this suggests that there might be different territorial effects produced by central government policies whose effectiveness varies from region to region. Many of the dimensions of well-being considered in the analysis (environment, health, education, essential public services, security) are indeed regulated by national policy programmes, yet the quality of services provided to citizens is ultimately uneven at the regional level. ${ }^{16}$ Moreover, regional and local authorities have played a key role in delivering public policies over the last few decades and the share of total national government spending accounted for by sub-national expenditure has grown (European Commission 2014).

Some interesting results for the overall well-being and per capita GDP arise out of the decomposition of the Theil index. First, the sharp difference in the trend of inequalities is worth noting when development is only looked at its economic dimension (per capita GDP) or when a multidimensional perspective (RWBI) is adopted. Indeed, disparities in terms of GDP, both within and between the sub-national macro-areas (North, Centre,

\footnotetext{
16 National spending budgets are transferred to regional governments on the basis of objective indicators, mainly socio-demographic and economic indices, under the constraint of achieving the social and financial goals set. For example, in the case of health care, since 2001 healthcare expenditure has been attributed to the regions under the constraints of achieving essential health care service levels (whose Italian acronym is LEA), set by the National government, and of observing the economic balance. Regions can provide additional healthcare services through their own resources, while regions with a deficit in health spending are subjected to sanctions by National government (higher co-payments by citizens, increased regional taxation) aimed at containing expenditure, and, in more serious cases, to the nomination of an external commissioner by the central government. For more details see Aimone Gigio et al. (2018) and Francese and Romanelli (2011).
} 
and Mezzogiorno), are stable over the whole period as shown by the flat lines in Fig. 2. The overall Theil index shows a decreasing trend when the analysis moves to the multidimensional well-being indicator, meaning that inequalities across Italian regions slightly decreased throughout the period whereas the within and the between components present different trends: the former exhibits a decreasing trend from 2006 onwards while the latter is characterised by peaks and troughs increasing in overall trend especially after 2010.

Furthermore, the decomposition clearly highlights that inequalities in GDP are entirely determined by disparities between sub-national macro-areas, as differences within the three areas approach zero. Conversely, the downward sloped pattern of the Theil index in terms of RWBI is mainly due to the within component of inequality, which shows a smooth decreasing trend, while the between component exhibits a fluctuating increasing tendency.

When each sub-national macro-area is considered separately, the highest level of inequalities for both GDP and RWBI indices is experienced by the Mezzogiorno area, where on average the Theil index is five times higher for RWBI and twenty times higher for percapita GDP compared to the North (Table 8 in "Appendix C"). Moreover, it should be noted that the evolution of inequalities over time follows different tendencies in the Italian sub-national macro-areas if the economic or the multidimensional well-being indicator is considered. The North experiences an increasing trend in the Theil index when it is calculated for GDP, yet the index has decreasing values when applied to the RWBI; Conversely, the opposite trend of GDP decreasing and the RWBI increasing occurs in the Mezzogiorno. In contrast, quite a stable trend for GDP and a decreasing tendency for the RWBI are found in the Central area.

Even though going into a far reaching investigation of the inequalities for each wellbeing dimension is beyond the main goals of this paper, it should be remembered that the definition of an overall index could hide a variety of dynamics which characterise the intermediate indices and, consequently, hide the multidimensional nature of well-being (Decancq et al. 2009). In particular, by looking at the evolution of the within and between components of inequalities dimension-by-dimension, the results can be summarised by identifying three different patterns regarding the components of the Theil Index (Table 7 in "Appendix C").

There is a first sub-set of well-being domains which confirm the existence of a sharp divide between northern, central and southern regions as shown by a prominence of the between-groups component of the Theil index: Culture and free time, Employment, Material conditions, Research and innovation, and Social relations and Health (except in 2007 and 2011 for the latter). What is more, the between-groups component increases over time for Culture and free time and Research and innovation whereas it decreases over time for Social relations and Employment. Conversely, for a second subset of dimensions, i.e. Education, Environment, and Personal security, the prevailing component is within-group inequality. This means that regions which fall into each sub-national macro-area are very dissimilar in terms of these well-being dimensions. The two components of the Theil Index only follow a fuzzier pattern for one dimension, i.e. Essential public services. Furthermore, similar to the findings obtained for RWBI and GDP, the Theil Index underpins higher inequalities in the Mezzogiorno area than in the rest of Italy regardless of the dimension considered. $^{17}$

\footnotetext{
17 More detailed results on the Theil's index for per capita GDP and RWBI and its sub-indicators are reported in the tables in Appendix $\mathrm{C}$ (Tables 7 and 8). In particular, Table 7 refers to the decomposition of the Theil index in the within and between components while, Table 8 shows the values for each subnational area.
} 

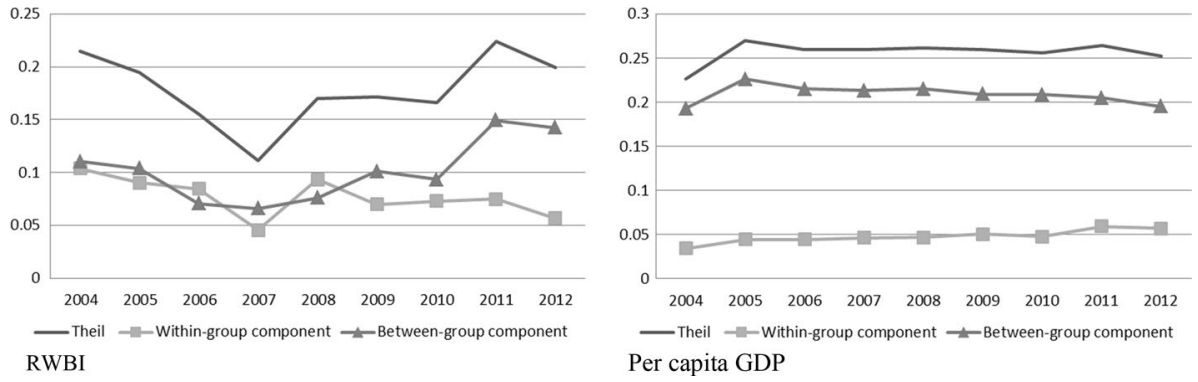

Fig. 2 Decomposition of the Theil index: between and within inequality. Source: Our elaboration on Istat and Ferrara and Nisticò (2015)

\section{Does Location Matter?}

The multifaceted picture of the development of Italian regions suggests that regional performance might also depend on the geographical proximity to other regions, i.e. spatial autocorrelation. In order to assess whether some spatial interactions were at work over the 2004-2012 period, we performed an exploratory spatial data analysis. The spatial association detects the existence of territorial clusters that do not necessarily overlap with the aggregations in the sub-national macro-areas.

In general, spatial correlation implies that two or more regions that are spatially close tend to be more similar to each other in terms of the attribute of interest than regions that are spatially distant (Anselin et al. 2008).

The following global Moran Index is adopted (Moran 1950):

$$
\mathrm{I}=\frac{1}{\mathrm{~s}^{2}} \frac{\sum_{\mathrm{i}} \sum_{\mathrm{j}}\left(\mathrm{y}_{\mathrm{i}}-\overline{\mathrm{y}}\right)\left(\mathrm{y}_{\mathrm{j}}-\bar{y}\right)}{\sum_{\mathrm{i}} \sum_{\mathrm{j}} \mathrm{w}_{\mathrm{ij}}}
$$

where $\mathrm{s}^{2}=\frac{\sum_{\mathrm{i}}\left(\mathrm{y}_{\mathrm{i}}-\overline{\mathrm{y}}\right)^{2}}{\mathrm{n}}$ is the sample variance, $y_{i}$ is the value of the variable of interest in area $i$ (either RWBI or per capita GDP in our case); $\bar{y}$ is the overall mean; $w_{i j}$ is the weight which defines proximity between $i$ and $j$, with $i \neq j$, and $n$ is the number of areal units considered. We consider row-standardized weights derived from an inverse distance measure.

Autocorrelation in well-being at the local level is detected by using LISA (Local Indicator Spatial Association), which is the decomposition of the global indicator into the contribution of each region. Therefore, we consider the local Moran Index (Ii) calculated for each areal unit:

$$
I_{i}=\frac{y_{i}-\bar{y}}{s} \sum_{j}^{n} W_{i j} \frac{\left(y_{j}-\bar{y}\right)}{s}
$$

when neighbouring regions have similar values, $I_{i}$ will be positive. Conversely, when they have different values, $I i$ will be negative.

Both at local and global level, when there is no correlation between neighbouring values,

$$
E(\mathrm{I})=-\frac{1}{\mathrm{n}-1}
$$


when $n$ tends to $\infty$, the expected value of I, $E(I)$, approaches 0 .

$I$ is asymptotically normally distributed, where:

$$
\frac{\mathrm{I}+\frac{1}{\mathrm{n}-1}}{\sqrt{\operatorname{Var}(\mathrm{I})}} \sim \mathrm{N}(0,1)
$$

The null hypothesis of absence of spatial association (i.e. $y_{i}$ iid) is tested by calculating the z-score:

$$
\mathrm{z}=\frac{\mathrm{I}-\mathrm{E}(\mathrm{I})}{\sqrt{\operatorname{Var}(\mathrm{I})}}
$$

We then compare the z-score to a standard normal distribution.

The test is performed to detect spatial correlation at both global and local level by considering the full sample and the usual three Italian sub-national areas (North, Centre, and Mezzogiorno). Hence, we calculate the Moran index for all the regions, finding evidence of a positive global spatial autocorrelation. The dynamics show that the index rises from 0.158 in 2004 to 0.285 in 2012 for overall well-being whereas it does not change significantly for GDP. A Moran scatterplot is displayed in Fig. 3.

Each quadrant in the scatterplot represents a different type of spatial autocorrelation whilst the slope of the line is Moran's I coefficient. What emerges from comparing panels (a) and (b) in Fig. 3 over time is a greater spatial association over time between the centre-northern regions clustered in quadrant I of the scatterplot, where the units reporting high levels of well-being associated with high levels of well-being in neighbours are grouped. In the specular section, regions that also have high levels of spatial association are found, yet they present low levels of well-being. It should be noted that regions in this quadrant are all from the South of Italy, but some of them (Campania, Calabria, and Sicily) are very strictly connected with each other in terms of both low levels of their own and the well-being of their neighbours, whilst others (Molise, Basilicata, Sardinia, and Abruzzo) do not exhibit evidence of spatial autocorrelation as the hypothesis of spatial independence is not rejected.

With regards to GDP, local spatial autocorrelation measurements depict a greater separation between northern and southern regions, especially in 2004. These latter lie closely in a cluster, which includes the Italian "less developed regions" as classified by the EU-Cohesion Policy 2014-2020 (Sicily, Campania, Calabria, Puglia, and Basilicata), both at the beginning and at the end of the study period (Fig. 4).

On the one hand, the results of the Local Indicator Spatial Association (LISA analysis) carried out in each of the three subnational areas (North, Centre, and Mezzogiorno) show evidence of local spatial independence for RWBI, thereby confirming that neighbouring regions may experience different patterns of well-being, which confirm the relevance of "within" disparities. On the other hand, the findings for GDP show a significant global spatial autocorrelation for the Mezzogiorno regions, underlining the predominance of "between" inequalities.

These results, providing evidence of spatial autocorrelation through the Moran index, suggest that a spatial component should be added to the model described in Sect. 3 .

First of all, in assessing the spatial dimension of the relationship analysed, it is crucial to decide which type of spatial interaction effects should be accounted for, whether 


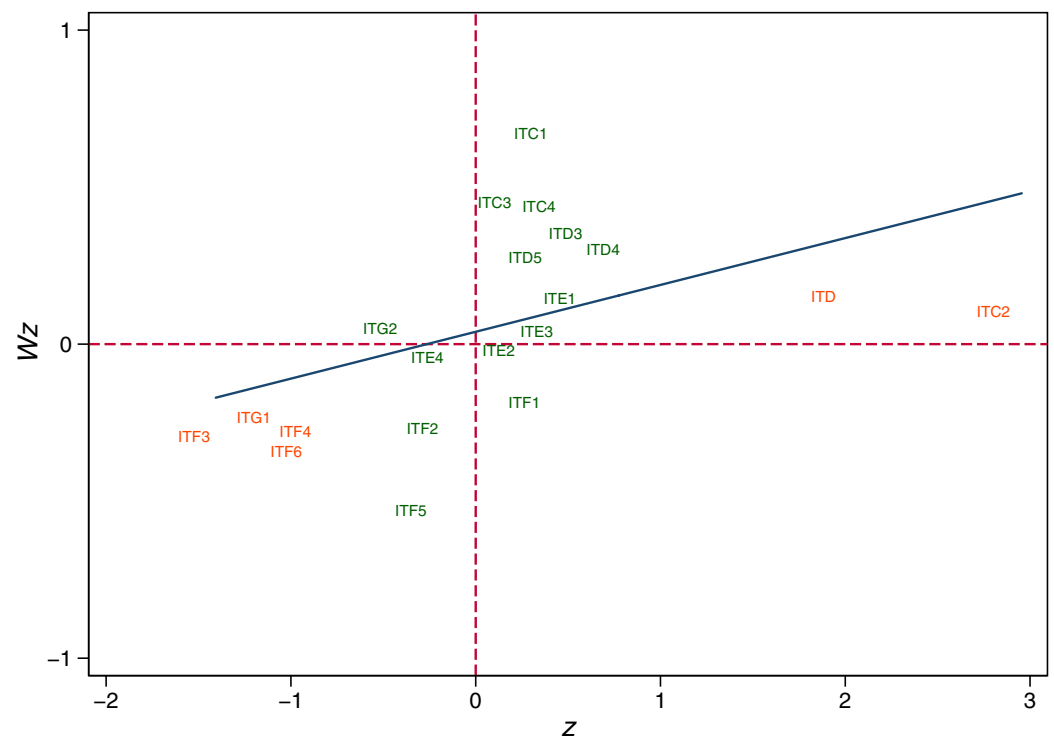

(a) 2004 - Moran's I $=0.149$ (p-val=0.000)

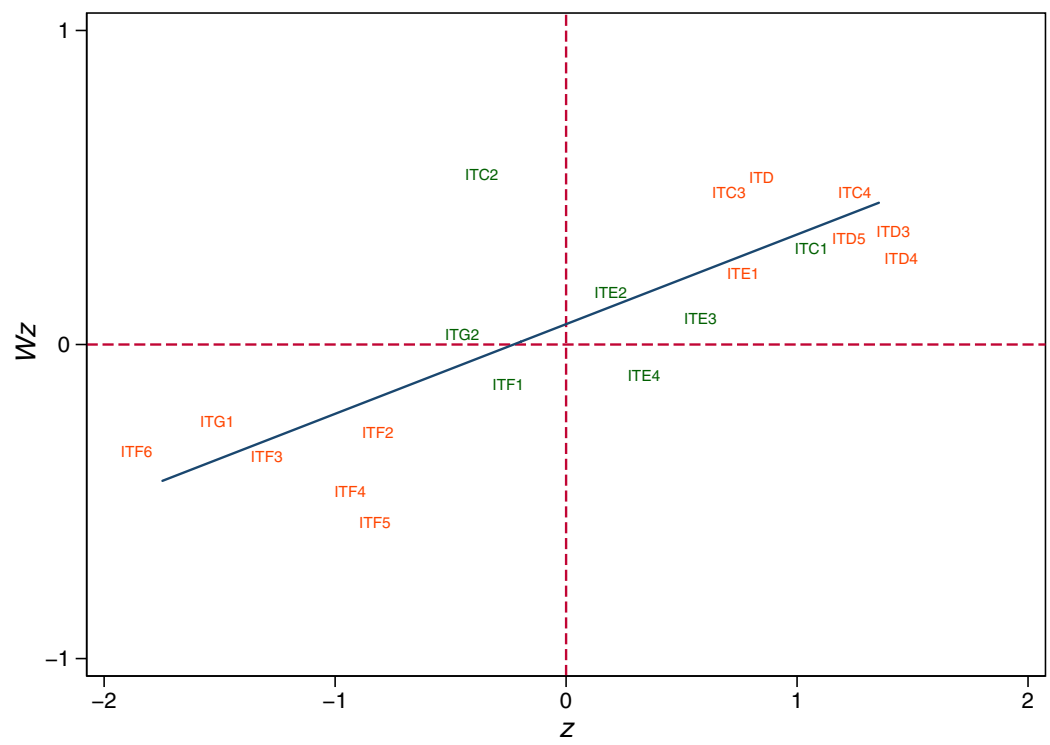

(b) 2012 - Moran's I $=0.285$ (p-val=0.000)

Fig. 3 Moran Index scatterplot for RWBI by Italian regions, years 2004 and 2012. Regional codes: ITF1Abruzzo, ITF5-Basilicata, ITF6-Calabria, ITF3-Campania, ITD5-Emilia-Romagna, ITD4-FriuliVenezia Giulia, ITE4-Lazio, ITC3-Liguria, ITC4-Lombardy, ITE3-Marche, ITF2_Molise, ITC1Piedmont, ITF4-Puglia, ITG2-Sardinia, ITG1 - Sicily, ITE1—Tuscany, ITD—Trentino, ITE2-Umbria, ITC2_-Valle d'Aosta, ITD3-Veneto. Source: our elaborations 


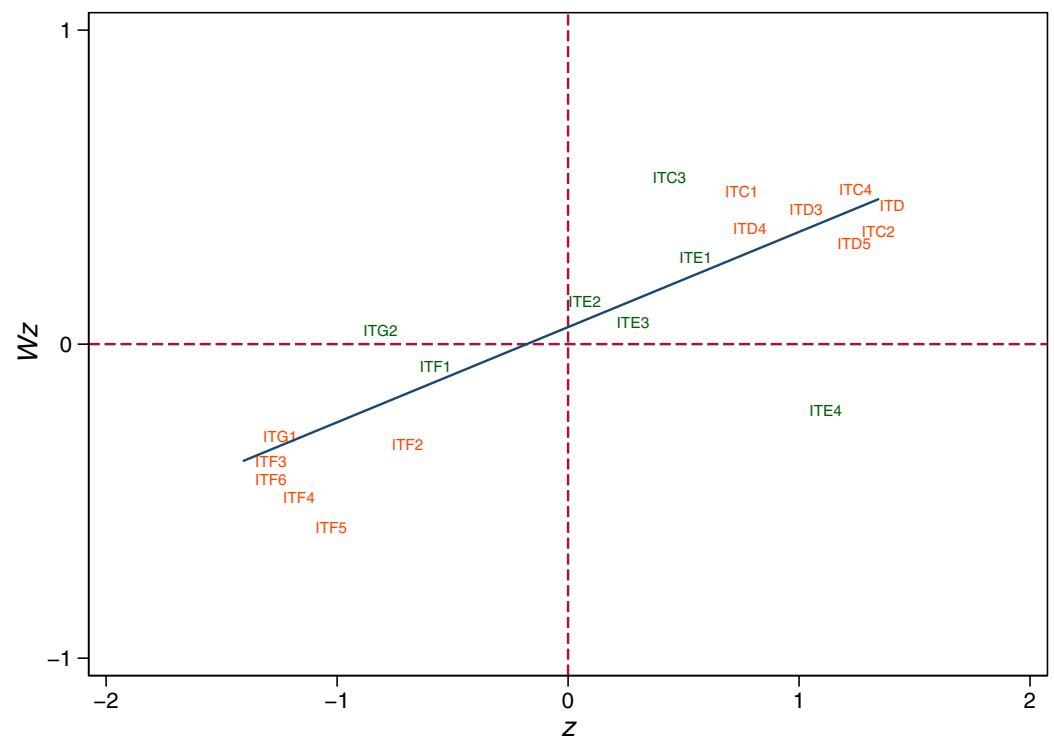

(a) 2004 - Moran's I $=0.303$ (p-val $=0.000$ )

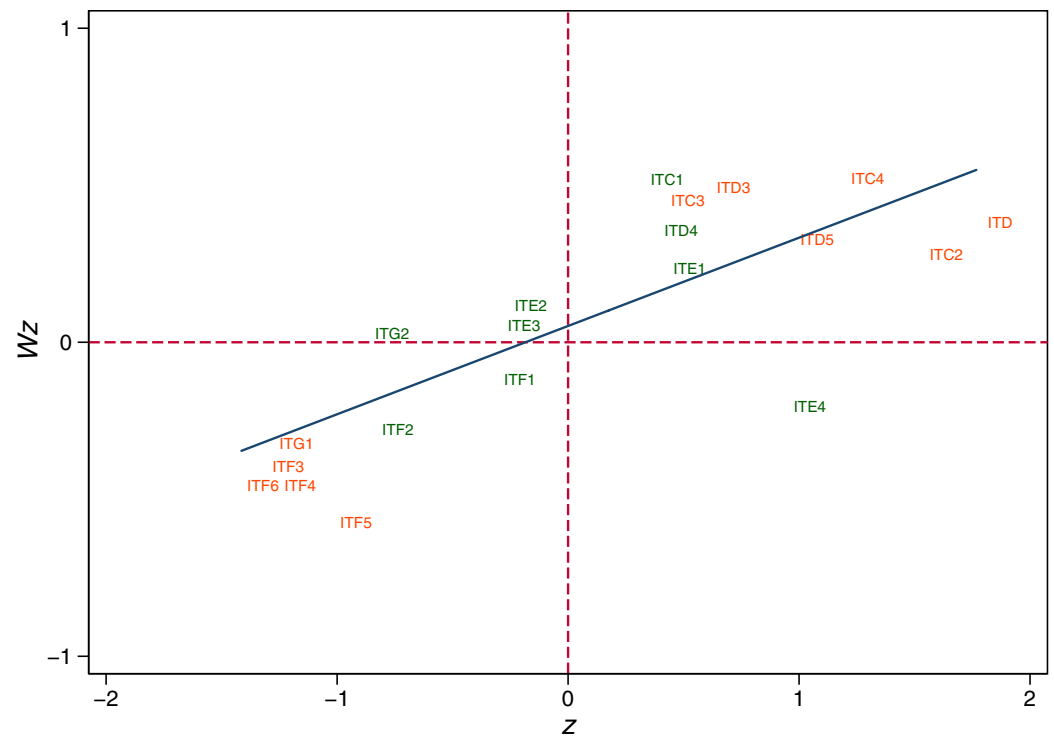

(b) 2012- Moran's I=0.281 (p-val $=0.000)$

Fig. 4 Moran Index scatterplot for per capita GDP by Italian regions, years 2004 and 2012. Regional codes: ITF1-Abruzzo, ITF5-Basilicata, ITF6-Calabria, ITF3-Campania, ITD5-Emilia-Romagna, ITD4Friuli-Venezia Giulia, ITE4-Lazio, ITC3-Liguria, ITC4-Lombardy, ITE3-Marche, ITF2-Molise, ITC1-Piedmont, ITF4-Puglia, ITG2-Sardinia, ITG1—Sicily, ITE1—Tuscany, ITD—Trentino, ITE2Umbria, ITC2 - Valle d'Aosta, ITD3-Veneto. Source: Our elaborations 
they should be spatial specific or time specific and whether they should be treated as fixed or random (Elhorst 2014). ${ }^{18}$

In order to choose the best spatial model specification, we follow the approach proposed by Elhorst (2014) and we refer to the general-to-specific approach of Lesage and Pace (2009).

For this purpose, we first estimate a Spatial Durbin Model (SDM) and then we test whether it can be simplified to a spatial lag (SAR) or a spatial error model (SEM).

The spatial regression equation used is:

$$
Y_{i, t}=\alpha+\rho W Y_{i, t}+\beta X+W X \theta++\varepsilon_{i, t}
$$

where $X$ is a vector of explanatory variables (IQI, per-capita expenditure in logarithm and their interaction term) and $W$ are the spatial weights extracted from a row-normalised binary contiguity matrix. Then we test whether either $\rho$ or $\theta$ are equal to zero or if there is an interaction among error terms, which may lead to a simplification of the SDM.

\section{Explaining Regional Differences in Well-Being with Institutional Quality}

In order to assess whether a better institutional context plays a significant role in explaining multidimensional regional well-being inequalities in Italy, we first estimate Eq. (1) with a panel fixed effects model (FE).

The choice of the FE model is supported by the Hausman test $\left(\chi^{2}=204.58\right.$ for $\Delta \mathrm{N}$-Rrwbi and $\chi^{2}=188.54$ for $\Delta \mathrm{A}$-Rrwbi, $p$ values $\left.=0.000\right)$ and the Mundlak (1978) test $\left(\chi^{2}=8.98, p\right.$ value $\left.=0.003\right)$ that point to the choice of a fixed effects rather than a random effects estimation. ${ }^{19}$ When considering the overall Institutional Quality Index, we found a positive effect on the mean deviation measure: the better the quality of institutions, the wider the differences between Italian regions (Table 1, col. 1 and col. 2). This is true for both the deviation at national and at local level, even though the latter is less precisely estimated ( $p$ value $=0.113$ ). Interestingly, when disentangling the effects for each of the IQI dimensions, the coefficient is positive for Voice, Rule of Law, and Regulatory but negative for Corruption and Government, even if results are only precisely estimated for Corruption and Regulatory (Table 1 columns 3 and 4), suggesting that a less corrupted environment may foster a reduction in regional multidimensional well-being disparities. Furthermore, the better the Regulatory quality, the wider the well-being differences are likely to be since an improvement in the regulatory quality may further enhance the differences between the leading and the lagging regions.

In other words, since the Corruption index assesses the reciprocity in the degree of gerrymandering in performing public functions, less manipulated government interventions may lead to a smoother and more equal development of regions, ceteris paribus.

\footnotetext{
18 A detailed review of spatial panels methods can be found in Elhorst (2014), Lesage and Pace (2009), and Anselin et al. (2008).

19 Even though this choice might appear counterintuitive, since institutional quality is a phenomenon varying weakly over time, the choice of a panel fixed effects model is also in line with Wooldridge (2010), who suggests using a random effects model only if the variation across entities is assumed to be random and uncorrelated with the independent variables included in the model. Moreover, he points out that the unobserved heterogeneity in a fixed effects framework is not treated as non-random, but is allowed to be arbitrarily correlated with the explanatory variables.
} 
Table 1 The relationship between institutional quality and regional well-being disparities: baseline model

\begin{tabular}{lllll}
\hline & $(1)$ & $(2)$ & $(3)$ & $(4)$ \\
& $\Delta \mathrm{N}-$ Rrwbi & $\Delta$ A-Rrwbi & $\Delta \mathrm{N}$-Rrwbi & $\Delta$ A-Rrwbi \\
\hline IQI & $0.538^{*}$ & 0.445 & & \\
Voice & $(0.274)$ & $(0.280)$ & & \\
& & & 0.582 & $0.991^{* * *}$ \\
Rule of Law & & $(0.397)$ & $(0.370)$ \\
& & 0.242 & 0.030 \\
Regulatory & & $(0.187)$ & $(0.163)$ \\
& & & $0.564^{* *}$ & 0.348 \\
Government & & $(0.231)$ & $(0.221)$ \\
& & -0.070 & 0.099 \\
Corruption & & $(0.212)$ & $(0.212)$ \\
& & $-0.365^{*}$ & -0.307 \\
FE & Yes & & $(0.219)$ & $(0.209)$ \\
Year dummies & Yes & Yes & Yes & Yes \\
Regional linear trend & Yes & Yes & Yes & Yes \\
Observations & 180 & Yes & Yes & Yes \\
$\mathrm{R}^{2}$ & 0.926 & 180 & 180 & 180 \\
\hline
\end{tabular}

This Table presents panel FE estimates of the relationship between institutional quality and regional wellbeing disparities. $I Q I_{i t}$ is the Institutional Quality Index defined by Nifo and Vecchione (2014); Rule of Law $_{i t}$, Regulatory ${ }_{i t}$, Government $i t$ and Corruption $i t$ are the IQI's sub-components; the dependent variable is a measure of deviation in terms of well-being, it is computed either as the difference between the national average RWBI in year $\mathrm{t}\left(\Delta \mathrm{N}-\mathrm{Rrwbi}_{i t}\right.$, col. 1 and 3$)$ or the sub-national RWBI mean value in year $\mathrm{t}\left(\Delta \mathrm{A}_{-\mathrm{Rrwbi}_{i t}}\right.$, col. 2 and 4) and the regional RWBI. All the models include regional and time fixed effects and a regional linear time trend. Robust standard errors are shown in parentheses. $* * *, * *$, and * indicate significance at the 1,5 , and $10 \%$ level, respectively

Conversely, Regulatory quality refers to the ability of a government to design and implement effective regulatory interventions so it is reasonable to argue that regions exhibiting higher values of regulatory quality are also those that probably have RWBI above the national (area) average and so a higher $(\Delta \mathrm{N}$-Rrwbi). This is also confirmed when the deviation for the subnational area mean value is computed, but Government exhibits a positive coefficient. However, it should be pointed out that deviation measured at macro area level might underestimate the analysed phenomenon since it only catches the differences of a region compared to similar regions (i.e. those in the same macro area, as also seen in the results of the exploratory spatial data analysis). Moreover, as subnational areas are a purely statistical classification and do not represent a legal administrative entity, restricting the comparison to the average of each subnational area might be a limitation.

Regional differences in well-being are undoubtedly related to regional expenditure in well-being domains. Accounting for regional expenditure, and for its joint effect with IQI, facilitates investigation of how differences in multidimensional regional well-being are affected by the heterogeneous level of institutional quality and heterogeneous levels of expenditure. Results for a dichotomous expenditure variable are summarized in Table 2. 
Table 2 The relationship between institutional quality and regional well-being disparities: heterogeneous effect

\begin{tabular}{|c|c|c|c|c|}
\hline & $\begin{array}{l}\text { (1) } \\
\Delta \mathrm{N} \text {-Rrwbi }\end{array}$ & $\begin{array}{l}\text { (2) } \\
\Delta \mathrm{A}-\text { Rrwbi }\end{array}$ & $\begin{array}{l}\text { (3) } \\
\Delta \mathrm{N}-\text { Rrwbi }\end{array}$ & $\begin{array}{l}\text { (4) } \\
\Delta \mathrm{A}-\text { Rrwbi }\end{array}$ \\
\hline \multirow[t]{2}{*}{ IQI } & 0.151 & 0.154 & & \\
\hline & $(0.200)$ & $(0.220)$ & & \\
\hline \multirow[t]{2}{*}{ High expenditure (HE) } & -0.098 & -0.005 & $-0.313^{* *}$ & -0.200 \\
\hline & $(0.081)$ & $(0.088)$ & $(0.130)$ & $(0.159)$ \\
\hline \multirow[t]{2}{*}{ IQI*HE } & $0.449 * * *$ & 0.273 & & \\
\hline & $(0.154)$ & $(0.165)$ & & \\
\hline \multirow[t]{2}{*}{ Voice } & & & $0.874 * *$ & $1.288^{* *}$ \\
\hline & & & $(0.358)$ & $(0.609)$ \\
\hline \multirow[t]{2}{*}{ Voice*HE } & & & 0.107 & -0.070 \\
\hline & & & $(0.279)$ & $(0.258)$ \\
\hline \multirow[t]{2}{*}{ Rule of Law } & & & 0.112 & -0.104 \\
\hline & & & $(0.195)$ & $(0.200)$ \\
\hline \multirow[t]{2}{*}{ Rule of Law*HE } & & & 0.147 & 0.127 \\
\hline & & & $(0.172)$ & $(0.184)$ \\
\hline \multirow[t]{2}{*}{ Regulatory } & & & $0.376^{*}$ & 0.168 \\
\hline & & & $(0.216)$ & $(0.217)$ \\
\hline \multirow[t]{2}{*}{ Regulatory*HE } & & & 0.250 & 0.200 \\
\hline & & & $(0.223)$ & $(0.192)$ \\
\hline \multirow[t]{2}{*}{ Government } & & & -0.061 & 0.105 \\
\hline & & & $(0.194)$ & $(0.231)$ \\
\hline \multirow[t]{2}{*}{ Government*HE } & & & 0.037 & -0.038 \\
\hline & & & $(0.214)$ & $(0.279)$ \\
\hline \multirow[t]{2}{*}{ Corruption } & & & $-0.557 * * *$ & $-0.489^{*}$ \\
\hline & & & $(0.179)$ & $(0.241)$ \\
\hline \multirow[t]{2}{*}{ Corruption*HE } & & & $0.258^{*}$ & $0.293^{* *}$ \\
\hline & & & $(0.143)$ & $(0.139)$ \\
\hline FE & Yes & Yes & Yes & Yes \\
\hline Year dummies & Yes & Yes & Yes & Yes \\
\hline Regional linear trend & Yes & Yes & Yes & Yes \\
\hline Observations & 180 & 180 & 180 & 180 \\
\hline $\mathrm{R}^{2}$ & 0.948 & 0.868 & 0.957 & 0.895 \\
\hline
\end{tabular}

This Table presents panel FE estimates of the relationship between institutional quality and regional wellbeing disparities. $I Q I_{i t}$ is the Institutional Quality Index defined by Nifo and Vecchione (2014); Rule of Law $_{i t}$, Regulatory ${ }_{i t}$, Government ${ }_{i t}$ and Corruption ${ }_{i t}$ are the IQI's sub-components; $H E_{i t}$ is a dummy variable equal to 1 if the region has expenditure (in well-being related domains) higher than the median value in year $t$; the dependent variable is a measure of deviation in terms of well-being, it is computed either as the difference between the national average RWBI in year $\mathrm{t}\left(\Delta \mathrm{N}-\mathrm{Rrwbi}_{i t}\right.$, col. 1 and 3$)$ or the sub-national RWBI mean value in year t ( $\triangle \mathrm{A}-\mathrm{Rrwbi}_{i}$, col. 2 and 4) and the regional RWBI. All the models include regional and time fixed effects and a regional linear time trend. Robust standard errors are shown in parentheses. $* * * * *$, and $*$ indicate significance at the 1,5 , and $10 \%$ level, respectively

Exhibiting a level of expenditure higher than the national median value for an average value of institutional quality is more likely to be associated with wider well-being disparities measured at both national and local level. In particular, when testing the joint 
significance of the IQI variable and the expenditure interaction term, the coefficient is equal to $0.6(p$ value $=0.005)$ for the national deviation measure and $0.427(p$ value $=0.072)$ for the local measure. Similarly, a stronger effect in both magnitude and significance is obtained when IQI sub-indices and expenditure are considered jointly. The same results are synthesized in Fig. 5.

Panel (a) of Fig. 5 refers to the cumulative effect of IQI and expenditure endowments on the deviation from the national mean in regions with expenditure lower than the median value whilst, panel (b) refers to those regions with expenditure above the median. ${ }^{20}$ IQI is taken at its mean value in both cases. This figure shows that expenditure endowments matter especially for Corruption, Regulatory quality, and Voice and accountability. In particular, the Corruption index plays a role in reducing regional multidimensional well-being disparities especially for lower expenditure levels; the coefficient is always negative, although it is lower in magnitude and less precisely estimated when expenditure is high. In line with the findings discussed above, the opposite is found for Regulatory quality which exhibits a positive coefficient and is estimated more precisely at high expenditure levels.

Expenditure as a continuous variable is also considered ${ }^{21}$ in order to exploit the synergies of IQI and expenditure in affecting multidimensional regional well-being inequalities more in detail. Each column of Table 3 identifies a certain expenditure level in correspondence with its percentiles $(5,50,75,90,95)$. Analogously, each row represents the same percentile for the institutional quality index. Any of the cells is the jointly estimated coefficient obtained when deriving the model equation in respect of IQI and allows the effect by each level of institutional quality and each level of expenditure to be disentangled. Results show that the magnitude of the coefficient is always increasing for higher levels of IQI and expenditure. Interestingly, the coefficient is negative-i.e., reduction of regional multidimensional well-being inequalities - for lower levels of expenditure (albeit not precisely estimated, $p$ value $=0.213$ ) and becomes positive when expenditure is higher than the median.

In other words, let us consider a region which has IQI close to the median value and low expenditure (e.g., Lazio in 2012), it could be likely to experience a reduction of the distance from the national average in terms of RWBI of about 0.178 . Conversely, another region with a similar value of IQI but a level of expenditure within the 90 percentile (e.g. Piedmont in 2009) could probably experience an increase in well-being differences of about 0.251 . This suggests that the regional institutional quality heterogeneously affects multidimensional well-being inequalities accordingly to the respective public expenditure levels.

The findings described above refer to non-spatial models which do not account for regional geographical proximity. On the contrary, Tables 4 and 5 show the results obtained when dealing with the spatial dimension. In particular, Table 4 presents the results of a Spatial Durbin Model with the outcome computed at both national (col. 1) and local level (col. 2).

Elhorst (2014) argues that a fixed effects model is more appropriate than a random one if the study area consists of all the regions in a country. This is also endorsed by the Hausman test $\left(\chi^{2}=24.26, p\right.$ value $=0.0010$ with the deviation measured at the national level and $\chi^{2}=12.14, p$ value $=0.0960$ for the measure at the macro-area level) which indicates the choice of a fixed effects model. So we adopt Quasi-Maximum Likelihood (QML)

\footnotetext{
20 See footnote 15 .

21 For the sake of brevity, we show the results only for the inequality measure defined at national level $(\Delta \mathrm{N}-\mathrm{Rrwbi})$.
} 
Fig. 5 The relationship between institutional quality and regional well-being disparities: difference between regions with high and low expenditure, by IQI components. Notes The figures report point estimates from the regression models presented in Table 2 (col. 3). 95th percent confidence intervals are also reported. Source: Our elaborations

estimators with both time and unit fixed effects. ${ }^{22}$ Following Lesage and Pace (2009) we start from the more general specification and then we carry out some tests, that is, we initially estimate the SDM and then we test for alternative simplified specifications to identify which model best suits the data.

The spatial spill-over effects in the SDM model (Table 4) are negative and significant for all of the variables, but positive for the interaction between IQI and expenditure. However, the $\rho$ coefficient is not statistically significant. Therefore, we test whether the SAR model would be a better option by testing if $\theta=0$ and $\rho \neq 0$, whilst if $\theta=-\beta \rho$, the best model would be the SEM.

In this case, all the tests suggest the choice of a SAR model $\left(\chi^{2}=6.45, p\right.$ value $=0.0398$, and $\chi^{2}=9.28$, $\mathrm{p}$ value $=0.0258$, respectively). Results are reported in Table 5 .

Findings provide evidence of some spatial interactions affecting regional multidimensional well-being disparities. Not surprisingly, the coefficient of the spatially lagged dependent variable $(\rho)$ has an opposite sign for each of the two dependent variables. By considering multidimensional well-being inequalities in terms of deviation at the macro area level, the comparison is implicitly restricted only to regions belonging to the same subnational area which might not necessarily overlap with the spatial contiguity matrix as regions belonging to different sub-national areas may also share a border. Column 1 in Table 5 shows the presence of positive spatial spillovers when computing inequality at the national level whereas the spatial coefficient of the SEM, column (3), is not significant. This finding suggests that regional multidimensional well-being inequalities vary accordingly to the level of multidimensional well-being disparities between geographical neighbours.

In other words, the model implies that a region's expected level of multidimensional well-being deviation from the national average would be 14.7 points lower if its neighbours had an average deviation equal to its minimum value (a leading region in terms of well-being level) compared with a neighbour average of 0 (equal to the national average). Conversely, a region with the maximum neighbour average value of deviation from the national mean (a region very lagging behind in terms of well-being) would be expected to be 14.7 points higher compared to a region with a neighbour average of zero. Adopting a measure of well-being inequality at the macro area level results in a different picture where a region geographically close to a leading region (lowest value of the deviation from the mean, even belonging to another area) might lead to higher differences in well-being compared to its own sub-national area than a region having a neighbour with RWBI equal to its area average (deviation equal to zero).

Overall, the results suggest that institutional quality affects multidimensional regional well-being inequalities heterogeneously according to both different government quality and regional expenditure endowments. However, regional differences in multidimensional well-being might be influenced by the geographical location and might benefit from the presence of spatial spillovers.

${ }^{22}$ The Stata xsmle routine developed by Belotti et al. (2017) is used. 


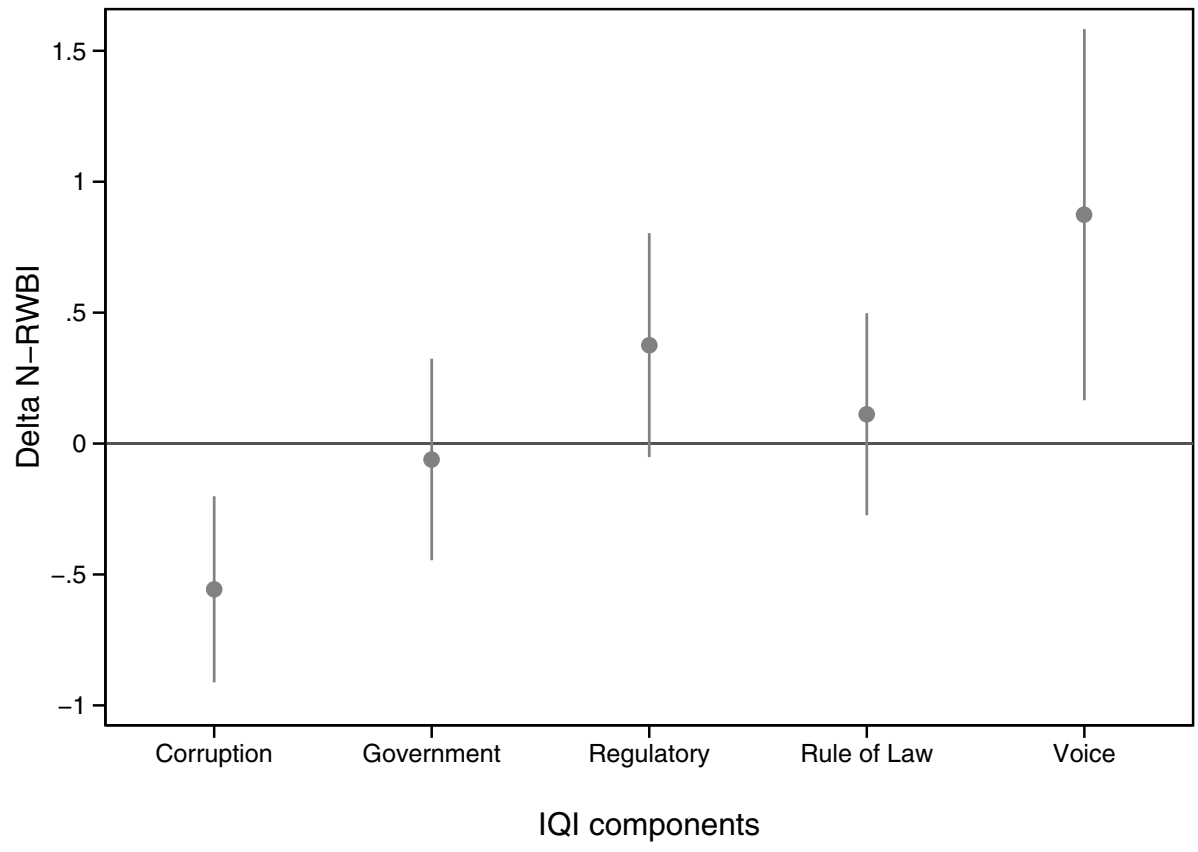

(a) Low expenditure

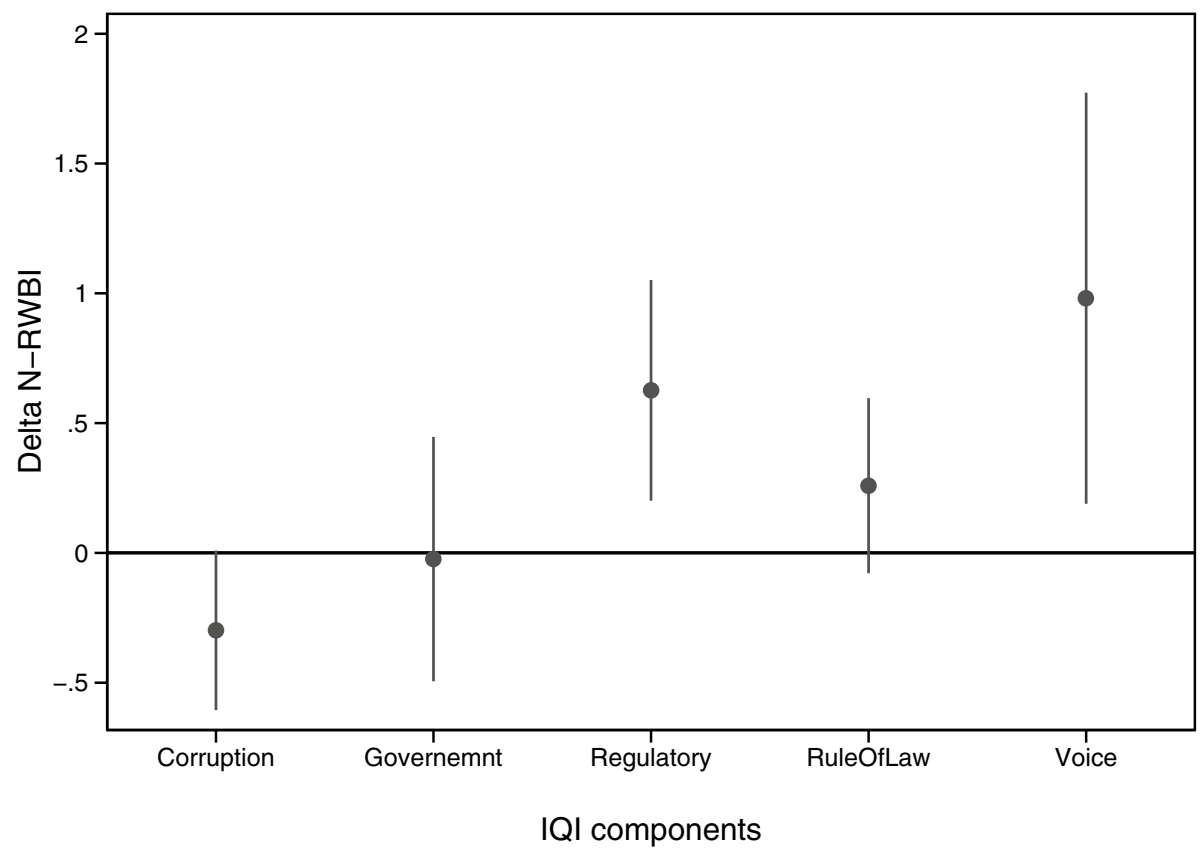

(b) High expenditure 
Table 3 The relationship between institutional quality and regional well-being disparities: heterogeneous effect with a continuous expenditure variable

\begin{tabular}{llllll}
\hline & $\begin{array}{l}\text { Expenditure } \\
\mathrm{p}(5)\end{array}$ & $\begin{array}{l}\text { Expenditure } \\
\mathrm{p}(50)\end{array}$ & $\begin{array}{l}\text { Expenditure } \\
\mathrm{p}(75)\end{array}$ & $\begin{array}{l}\text { Expenditure } \\
\mathrm{p}(90)\end{array}$ & Expenditure $\mathrm{p}(95)$ \\
\hline IQI p(5) & -0.049 & 0.049 & $0.056^{*}$ & $0.068^{* *}$ & $0.106^{* * *}$ \\
& $(0.039)$ & $(0.032)$ & $(0.032)$ & $(0.033)$ & $(0.035)$ \\
IQI p(50) & -0.178 & 0.181 & $0.206^{*}$ & $0.251^{* *}$ & $0.389^{* * *}$ \\
& $(0.143)$ & $(0.118)$ & $(0.118)$ & $(0.119)$ & $(0.128)$ \\
IQI p(75) & -0.194 & 0.197 & $0.224^{*}$ & $0.273^{* *}$ & $0.423^{* * *}$ \\
& $(0.155)$ & $(0.128)$ & $(0.129)$ & $(0.130)$ & $(0.140)$ \\
IQI p(90) & -0.217 & 0.220 & $0.250^{*}$ & $0.305^{* *}$ & $0.472^{* * *}$ \\
& $(0.173)$ & $(0.143)$ & $(0.144)$ & $(0.145)$ & $(0.156)$ \\
IQI p(95) & -0.233 & 0.237 & $0.269^{*}$ & $0.328^{* *}$ & $0.508^{* * *}$ \\
& $(0.187)$ & $(0.154)$ & $(0.154)$ & $(0.156)$ & $(0.168)$ \\
\hline
\end{tabular}

This Table presents panel FE estimates of the relationship between institutional quality and regional wellbeing disparities. $I Q I_{i t}$ is the Institutional Quality Index defined by Nifo and Vecchione (2014); the dependent variable is a measure of deviation in terms of well-being, it is computed as the difference between the national average RWBI in year $\mathrm{t}\left(\Delta \mathrm{N}-\mathrm{Rrwbi}_{i t}\right)$ and the regional RWBI. The columns refers to the percentiles of the $\ln (\text { Expenditure })_{i t}$, whilst the rows are the percentiles of $I Q I_{i t}$. Each cell identifies the joint effect of IQI and its interaction with the expenditure term. The model includes regional and time fixed effects and a regional linear time trend. Robust standard errors are shown in parentheses. ${ }^{* *},{ }^{* *}$, and $*$ indicate significance at the 1,5 , and $10 \%$ level, respectively

\section{Conclusions}

There is widespread agreement on the importance of the role played by institutions in shaping the development at different levels (countries, regions, individuals) and in several aspects beyond the strictly economic sphere. A similar extensive consensus has gained ground in promoting the idea that GDP is not an exhaustive measure of well-being. Both research flows claim that the phenomena have a multidimensional nature. Even though they are strongly linked, since institutional quality has been recognised as a driver of development and, in turn, of multidimensional well-being, literature assessing their respective relationship is still lacking. The assessment of regional well-being inequalities in relation to the quality of the institutions is the strand of research where this paper strives to make a contribution. Italy, historically characterised by pronounced regional gaps, has offered fruitful insights for the purposes of this analysis.

The decomposition of the Theil index has highlighted marked differences between well-being and GDP in terms of both the between and within components of the inequality indices, confirming that well-being is not simply a matter of GDP dynamics and that much more may be learned from non-productive spheres, especially regarding the nature, entity, and evolution of territorial disparities. Moreover, explaining inequalities in well-being is even more complex than interpreting the historical productive divide in Italy given that quality-of-life sub-indices move in conflicting ways.

The assessment of regional multidimensional well-being dynamics has also drawn attention on the spatial component of inequalities, confirming that there is evidence of some spatial spillovers in multidimensional well-being making Italian regions not independent from the performance of their neighbours. 
Table 4 The relationship between institutional quality and regional well-being disparities: Spatial Durbin model

\begin{tabular}{lll}
\hline & $(1)$ & $(2)$ \\
& $\Delta \mathrm{N}-$ Rrwbi & $\Delta$ A-Rrwbi \\
\hline IQI & $-3.188^{* * * *}$ & $-1.743^{* * * *}$ \\
& $(0.490)$ & $(0.446)$ \\
$\ln ($ Expenditure) & $-0.096^{* * *}$ & 0.002 \\
& $(0.037)$ & $(0.033)$ \\
IQI*In(Expenditure) & $0.420^{* * *}$ & $0.241^{* * *}$ \\
& $(0.056)$ & $(0.051)$ \\
$\mathrm{W}^{*} \mathrm{IQI}$ & $-2.469^{* *}$ & -0.735 \\
& $(0.995)$ & $(0.829)$ \\
$\mathrm{W}^{*} \ln ($ Expenditure) & $-0.226^{* *}$ & -0.058 \\
& $(0.090)$ & $(0.077)$ \\
$\mathrm{W}^{*} \mathrm{IQI}{ }^{*} \ln ($ Expenditure) & $0.287^{* *}$ & 0.100 \\
& $(0.128)$ & $(0.107)$ \\
$\rho$ & 0.075 & $-0.348^{* * * *}$ \\
FE & $(0.095)$ & $(0.114)$ \\
Year dummies & Yes & Yes \\
Spatial model & Yes & Yes \\
Observations & $\mathrm{SDM}$ & $\mathrm{SDM}$ \\
$\mathrm{R}^{2}$ & 180 & 180 \\
Number of regions & 0.157 & 0.005 \\
\hline
\end{tabular}

This table presents spatial panel Static Durbin Model (SDM) FE estimates of the relationship between institutional quality and regional well-being disparities. $I Q I_{i t}$ is the Institutional Quality Index defined by Nifo and Vecchione (2014); $\ln \left(\right.$ Expenditure $_{i t}$, is the natural log of the regional per-capita expenditure in wellbeing related domains; $W$ are the weights extracted from a row-normalised contiguity matrix; the dependent variable is a measure of deviation in terms of well-being, it is computed either as the difference between the national average RWBI in year $\mathrm{t}\left(\Delta \mathrm{N}-\mathrm{Rrwbi}_{i}\right.$, col. 1$)$ or the sub-national RWBI mean value in year $\mathrm{t}$ $\left(\triangle \mathrm{A}-\mathrm{Rrwbi}_{i}\right.$, col. 2) and the regional RWBI. All the models include regional and time fixed effects. Robust standard errors are shown in parentheses. $* * *, * *$, and * indicate significance at the 1,5 , and $10 \%$ level, respectively

Other than exploring the pattern of the spatial autocorrelation, the analyses focused on the role it played in affecting the interaction between multidimensional well-being inequalities and quality of government.

The main message this paper may help to deliver is that institutional quality affects regional multidimensional well-being inequalities. However, it would be too naive to assume that this relation is independent of any other factor. Results suggest that there is some heterogeneous effect of institutional quality on well-being inequalities, varying accordingly to the level of expenditure, to the institutional dimension considered, and the geographical location of the region.

From a more policy-oriented perspective, policymakers should ensure all citizens have the same chance of benefitting from tangible and intangible public goods, which ideally requires all of the regions to be close to the national well-being and GDP average. However, this goal calls for the design and implementation of sounder 
Table 5 The relationship between institutional quality and regional well-being disparities: Spatial Lag model and Spatial Error Model

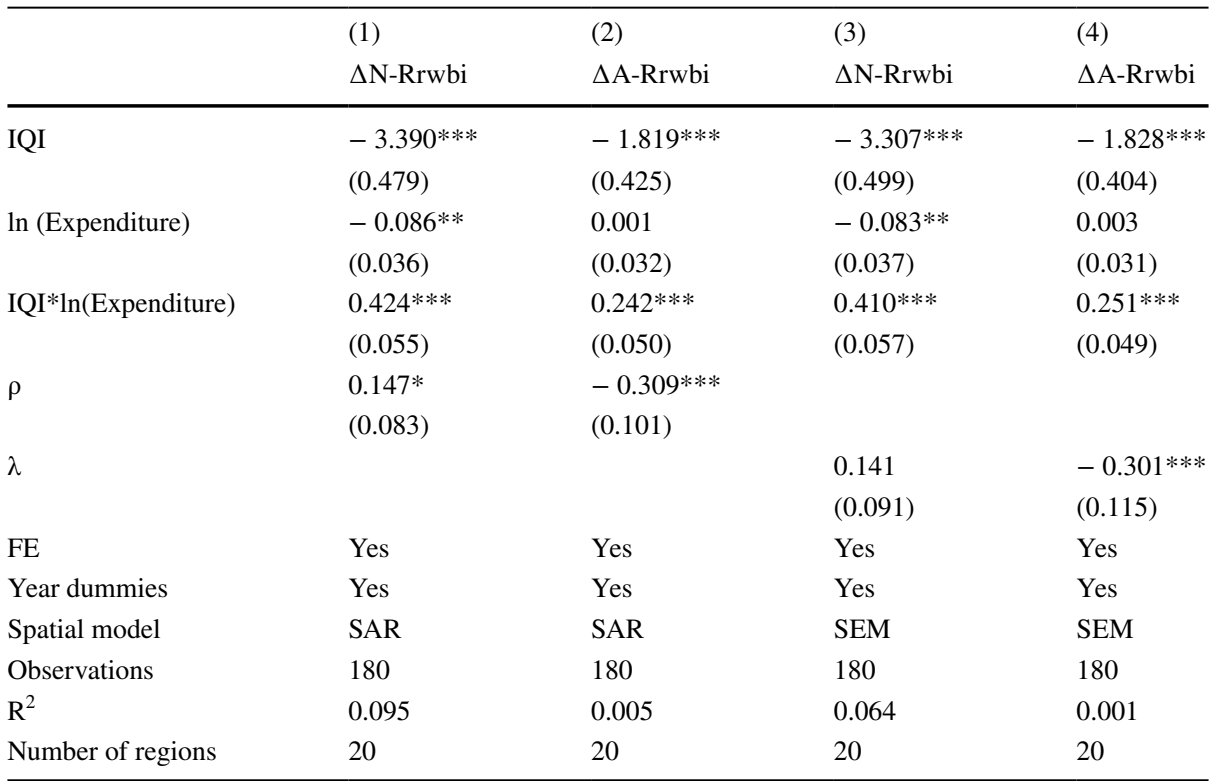

This table presents spatial panel FE estimates, Spatial Lag Model (SAR) and Spatial Error Model (SEM) of the relationship between institutional quality and regional well-being disparities. $I Q I_{i t}$ is the Institutional Quality Index defined by Nifo and Vecchione (2014); $\ln \left(\right.$ Expenditure $_{i t}$, is the natural $\log$ of the regional per-capita expenditure in well-being related domains; $\rho$ and $\lambda$ are the spatial terms; the dependent variable is a measure of deviation in terms of well-being, it is computed either as the difference between the national average RWBI in year $\mathrm{t}\left(\Delta \mathrm{N}-\mathrm{Rrwbi}_{i t}\right.$, col. 1 and 3$)$ or the sub-national RWBI mean value in year $\mathrm{t}$ $\left(\Delta \mathrm{A}-\mathrm{Rrwbi}_{i t}\right.$, col. 2 and 4$)$ and the regional RWBI. All the models include regional and time fixed effects. Robust standard errors are shown in parentheses. $* * *, * *$, and * indicate significance at the 1,5 , and $10 \%$ level, respectively

policy actions, targeted on both fostering more effective interventions especially in the lagging regions, and on enhancing overall well-being and economic development. The analyses helped to disentangle how expenditure endowments and different institutional quality domains might differently affect Italian regional inequalities in well-being. However, the overall message is not easy to read as higher expenditure levels are not always paired with lower inequalities, but several factors might play a role. In particular, expenditure should be flanked by an efficient regulatory quality to ensure policy interventions are effective. In this context, corruption is a factor that cannot be discarded. The results confirm that a less corrupted environment may favour the reduction of multidimensional well-being inequalities; however, the effect is partially mitigated when expenditure is higher, as some negative externalities might be at work. Additionally, this already complex picture is further complicated by the spatial spillovers that affect regional differences in multidimensional well-being.

Overall, our findings suggest that an adequate governance system could lead to lower regional multidimensional well-being inequalities, i.e. regions experiencing similar quality-of-life conditions, only if it is combined with better targeted local policies and with an efficient expenditure structure able to tackle regional weaknesses. 
Acknowledgements The authors would like to thank the editor and two anonymous reviewers for the insightful comments on an earlier version of the manuscript. Any errors are our own. The scientific output expressed does not imply a policy position of the European Commission. Neither the European Commission nor any person acting on behalf of the Commission is responsible for the use that might be made of this publication.

Open Access This article is distributed under the terms of the Creative Commons Attribution 4.0 International License (http://creativecommons.org/licenses/by/4.0/), which permits unrestricted use, distribution, and reproduction in any medium, provided you give appropriate credit to the original author(s) and the source, provide a link to the Creative Commons license, and indicate if changes were made.

\section{Appendix A: RWBI and IQI}

See the Figs. 6, 7 and 8 .
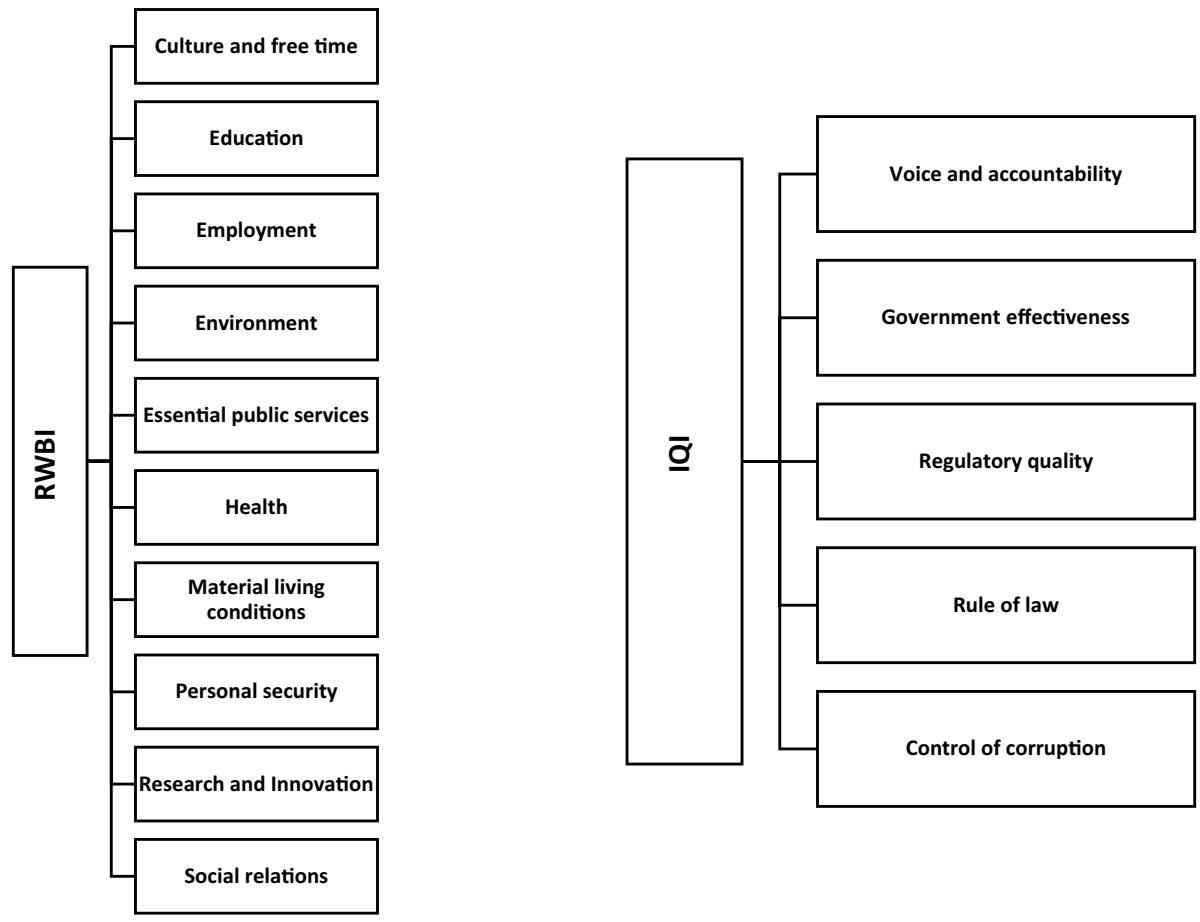

Fig. 6 The Regional Well-Being Index (RWBI) and the Institutional Quality Index (IQI). Source: Ferrara and Nisticò (2015); Nifo and Vecchione (2014) 


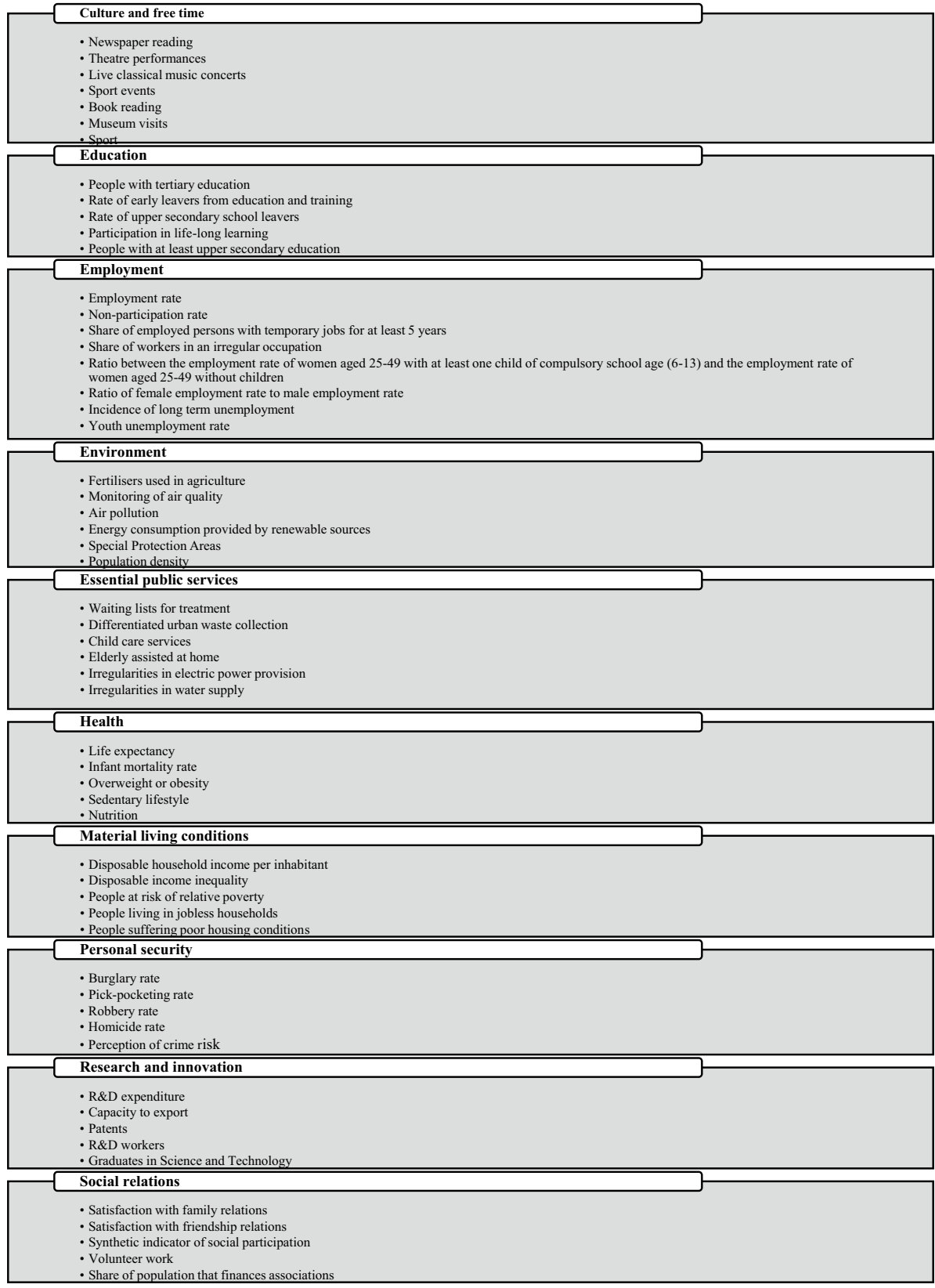

Fig. 7 Regional Well-Being Index (RWBI) dimensions and indicators. Source: Ferrara and Nisticò (2015) 


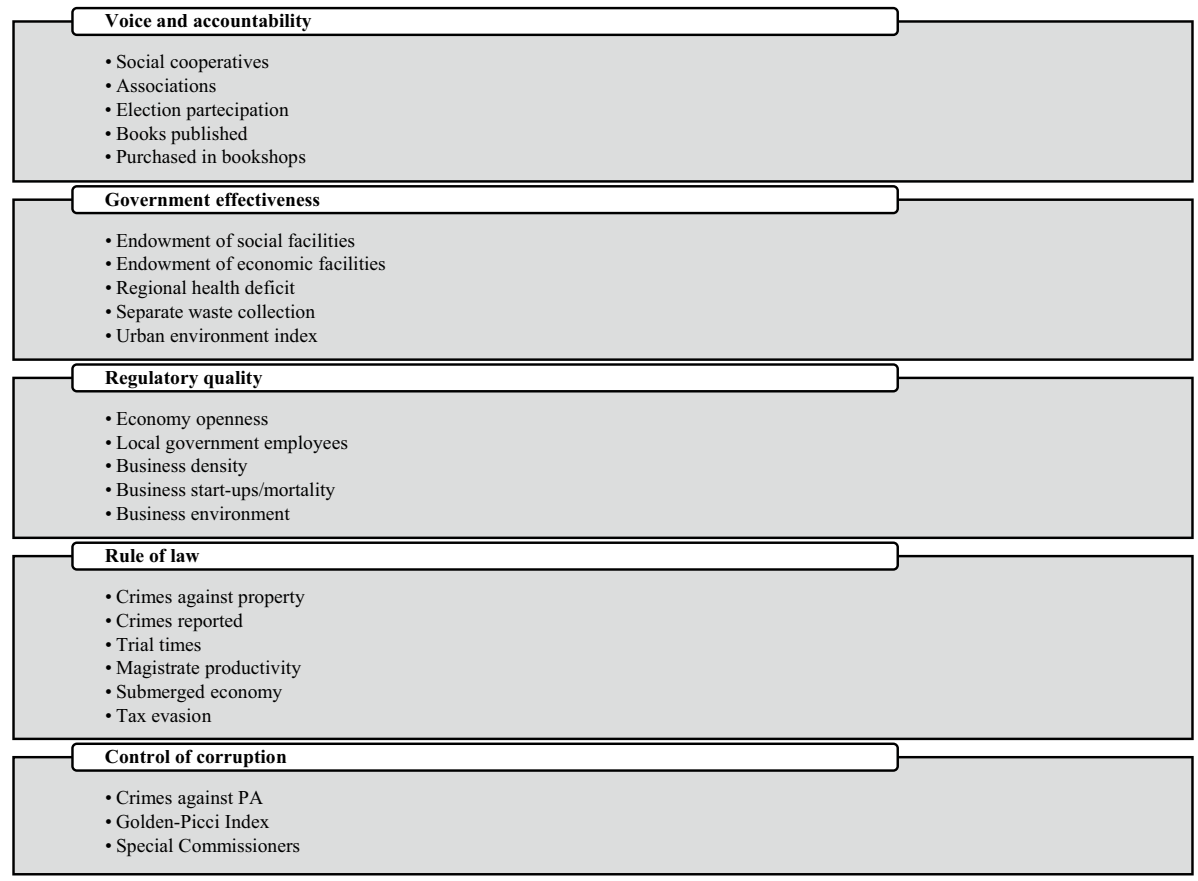

Fig. 8 Institutional Quality Index (IQI) dimensions and indicators. Source: Nifo and Vecchione (2014)

\section{Appendix B: The Relationship Between GDP, IQI and RWBI}

The relationship between RWBI and the IQI index is plotted in a bivariate scatterplot (Fig. 9). The figure shows a positive relationship between well-being levels and the IQI. Only four southern regions (Calabria, Campania, Sicilia and Puglia) are located on the low left side of the graph, far apart from the others (Abruzzo, Basilicata, Molise and Sardinia), which are closer to the group of the centre-northern regions. These latter are clustered in the upper-right side of the scatterplot, albeit there are two stand-alone regions (Valle d'Aosta and TrentinoAlto Adige) in the upper-right corner of the graph.

Whereas, Fig. 10 shows the within (macro-area) regional differences in well-being (panel a), IQI (panel b) and per capita GDP (panel c) using a box-plot graph: it highlights the existence of marked "between" divergences as the maximum value of the RWBI and per capita GDP in the Mezzogiorno area is slightly lower than the respective minimum values of the North. Furthermore, for the IQI the maximum value of the Mezzogiorno is below the median value of the North.

The length of the segment between the minimum and the maximum values of each box measures the "within" differences and it is much bigger in the South compared to the North and the Centre for the RWBI and the IQI. Conversely when the normalised per capita GDP is considered, "within differences" are wider in the North than in the South.

See the Table 6. 


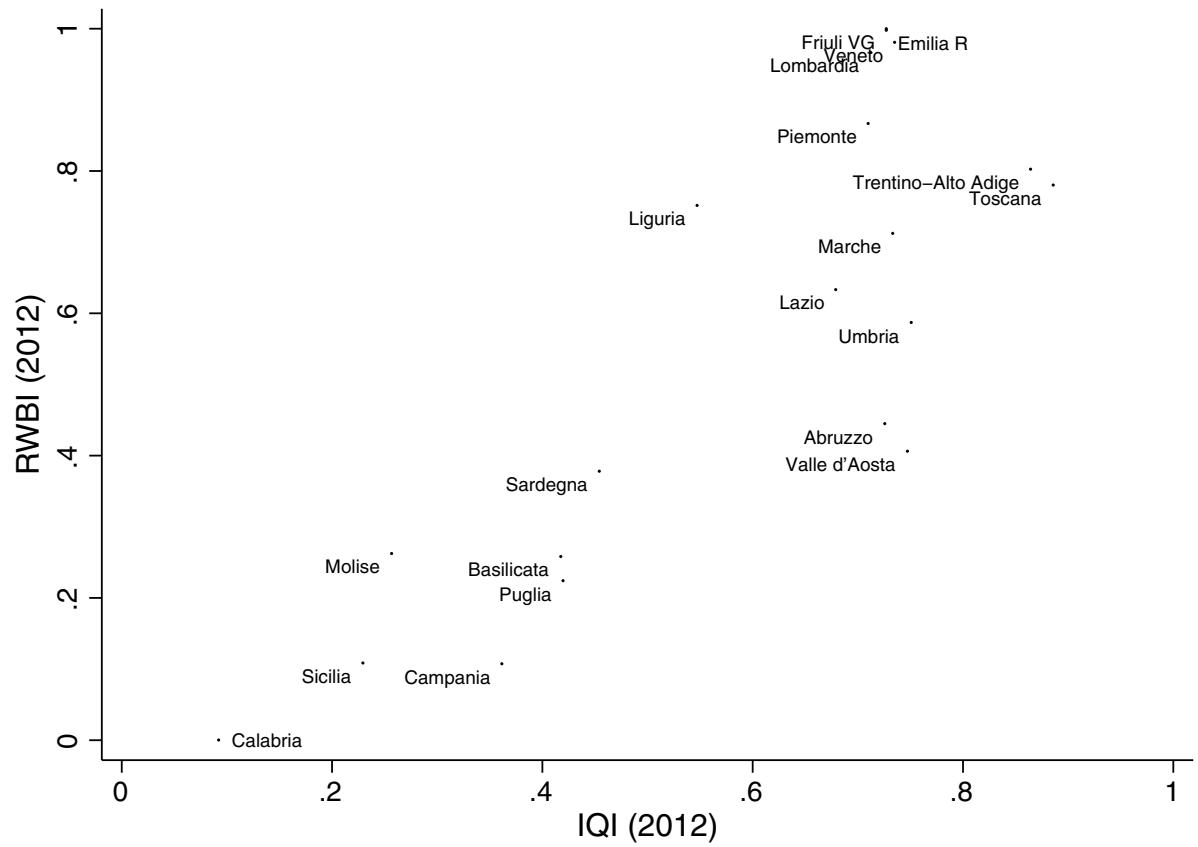

Fig. 9 Well-being and institutional quality index by region. Source: Our elaborations on Ferrara and Nisticò (2015) and Nifo and Vecchione (2014) data

\section{Appendix C: Methodological Notes on Theil's Index Decomposition}

The Theil index is a special case of the generalised entropy measure for which the sensitivity parameter-the parameter which determines the weight assigned to the upper tail—is set to 1 . For the Theil index, all the regions have the same weight independently of their level of wellbeing (Cowell 2011):

$$
T^{(t)}=\frac{1}{n} \sum_{i=1}^{n} \frac{I_{i}^{(t)}}{\mu\left(I^{(t)}\right)} \log \left(\frac{I_{i}^{(t)}}{\mu\left(I^{(t)}\right)}\right)
$$

The inequality within $\left(T^{W}\right)$ is the weighted average of the inequality within each area, whereas the inequality between $\left(T^{B}\right)$ represents the level of inequality there would be if there were no differences within areas. Formally:

$$
\begin{array}{r}
T^{B}=\sum_{r=1}^{3} s_{r}\left(\frac{\mu_{r}}{\mu_{T}}\right) \log \left(\frac{\mu_{r}}{\mu_{T}}\right) \\
T^{W}=\sum_{r=1}^{3} s_{r}\left(\frac{\mu_{r}}{\mu_{T}}\right) T_{r}
\end{array}
$$

where $s_{r}$ is the proportion of regions included in the sub-national area $r, \mu_{r}$ is the mean of the sub-national area $r$ and $\mu_{T}$ is the global mean of the variable under study. Finally, $\mathrm{T}_{\mathrm{r}}$ is the Theil index of the sub-national area.

See the Tables 7 and 8 . 


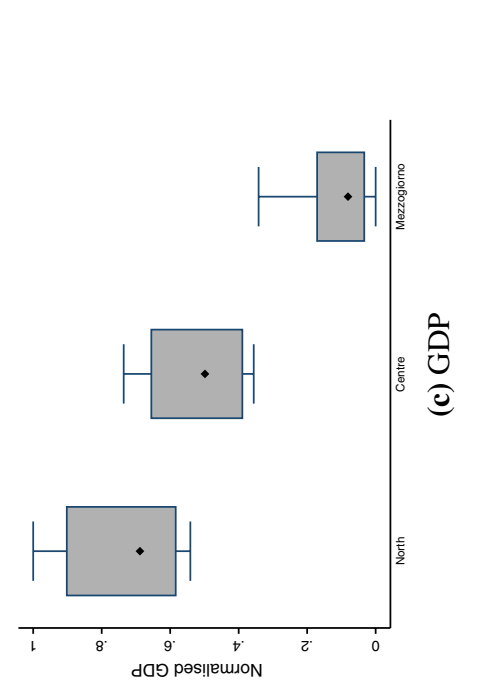

$\ddot{\check{\Xi}}$

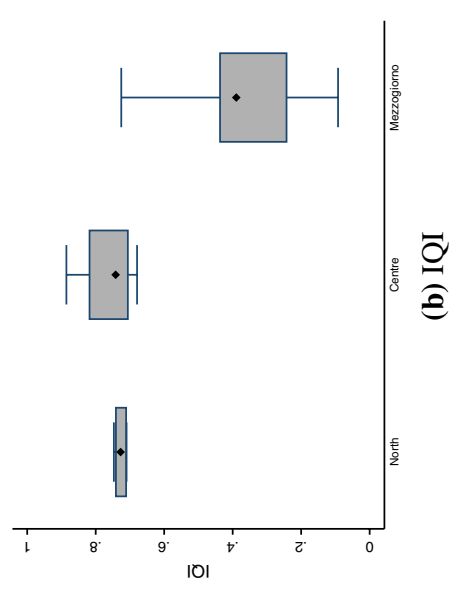

๐ั่

官

$\frac{1}{80}$

更

$\stackrel{\Xi}{\Xi}$

తే

芒

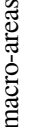

స్ّ

ลे

จิ

퓰 중

.

紊菊

్ㅗ 모

ชै

:

응

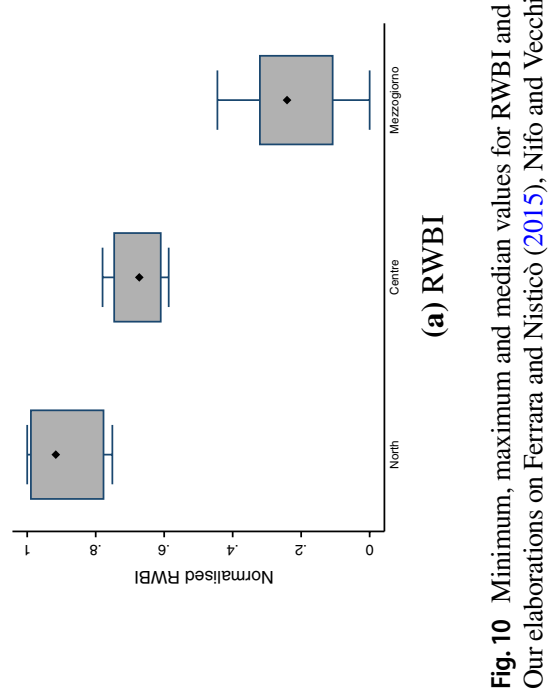




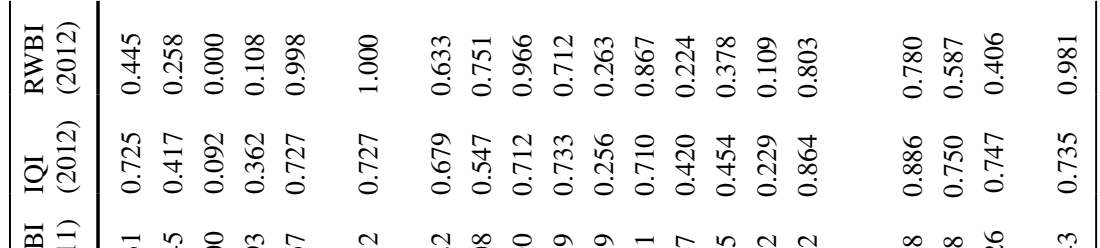

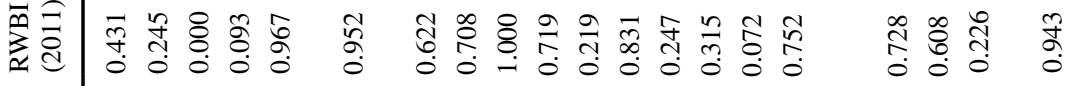

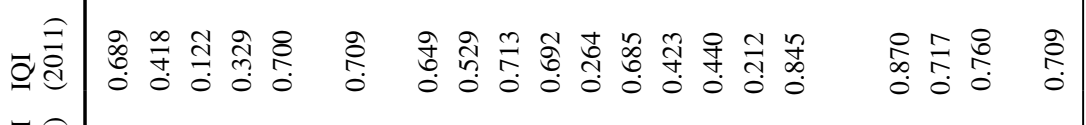

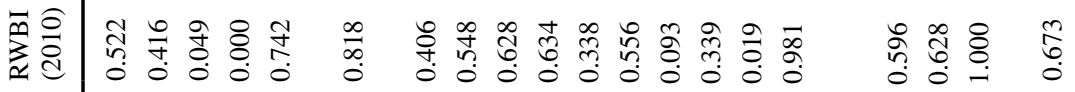

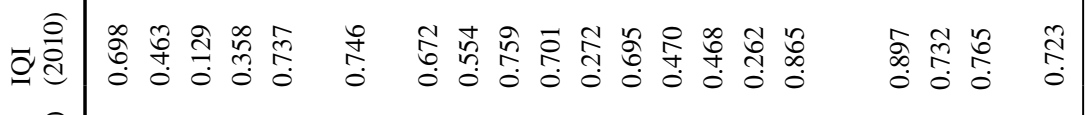

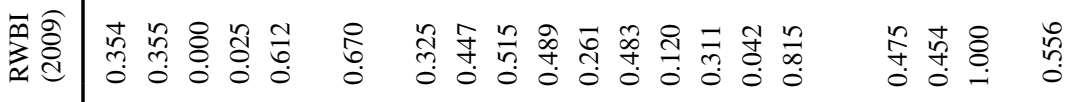

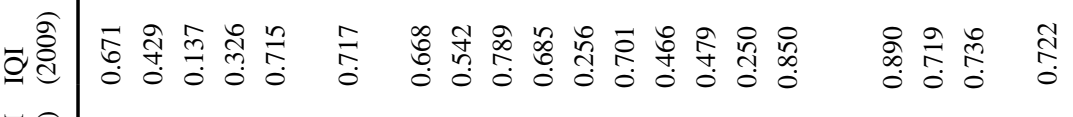

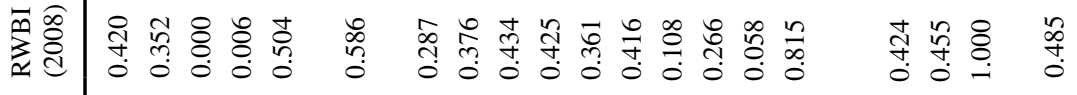

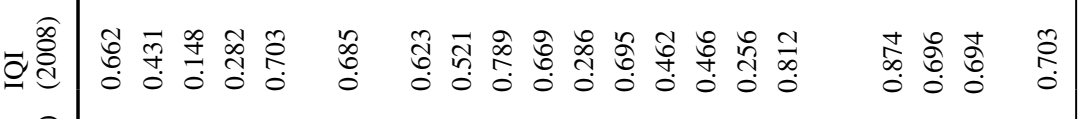

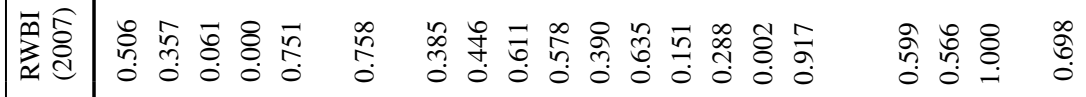

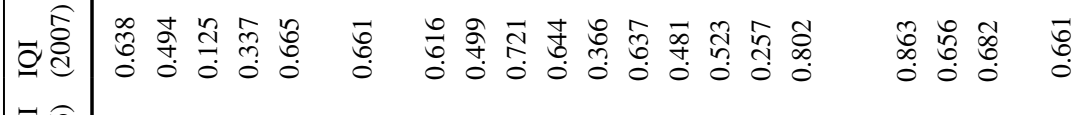

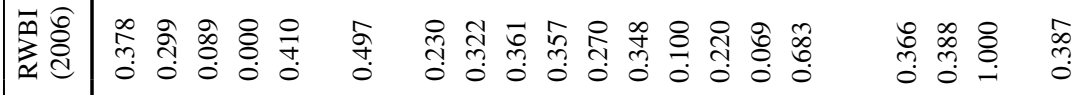

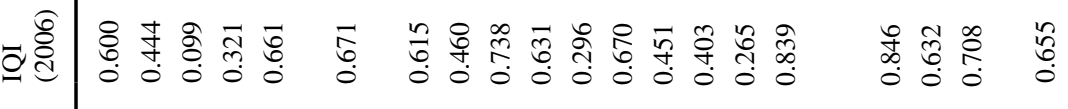

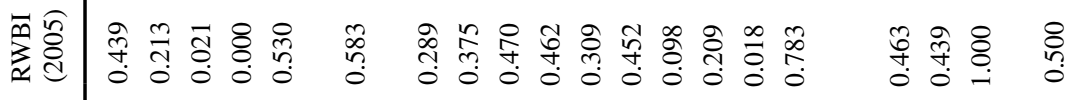

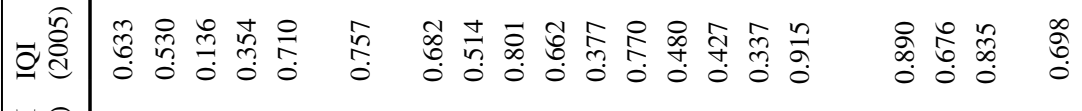

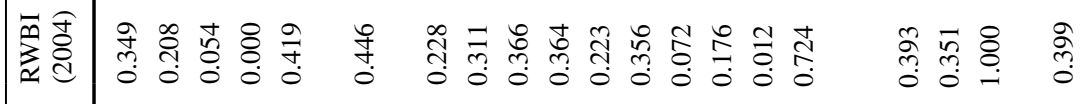

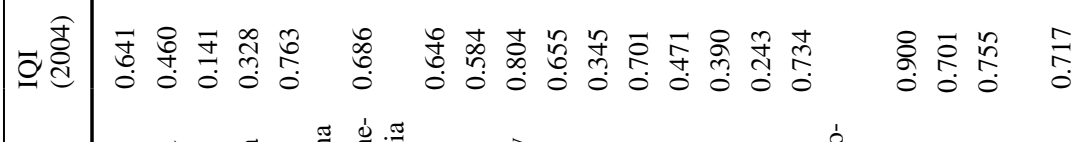

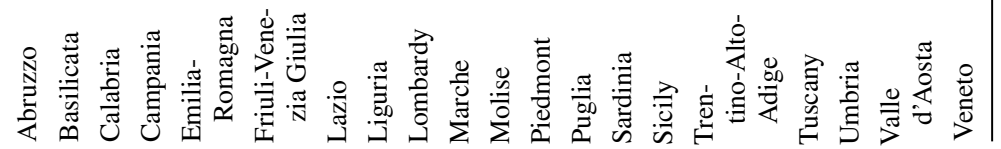

กิ

:

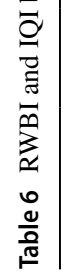


Table 7 Decomposition of the Theil index: between and within inequality. Source: Our elaborations on Ferrara and Nisticò (2015) and Istat data

\begin{tabular}{|c|c|c|c|c|c|c|c|c|c|}
\hline & 2004 & 2005 & 2006 & 2007 & 2008 & 2009 & 2010 & 2011 & 2012 \\
\hline \multicolumn{10}{|c|}{ Culture and free time } \\
\hline Theil & 0.138 & 0.208 & 0.190 & 0.183 & 0.226 & 0.214 & 0.218 & 0.198 & 0.318 \\
\hline Within & 0.033 & 0.066 & 0.053 & 0.046 & 0.065 & 0.060 & 0.065 & 0.061 & 0.067 \\
\hline Between & 0.105 & 0.142 & 0.137 & 0.137 & 0.161 & 0.155 & 0.153 & 0.137 & 0.250 \\
\hline \multicolumn{10}{|c|}{ Education } \\
\hline Theil & 0.231 & 0.191 & 0.065 & 0.094 & 0.070 & 0.062 & 0.129 & 0.085 & 0.090 \\
\hline Within & 0.170 & 0.157 & 0.046 & 0.086 & 0.066 & 0.056 & 0.122 & 0.079 & 0.090 \\
\hline Between & 0.061 & 0.034 & 0.019 & 0.009 & 0.005 & 0.006 & 0.007 & 0.006 & 0.000 \\
\hline \multicolumn{10}{|c|}{ Employment } \\
\hline Theil & 0.137 & 0.107 & 0.125 & 0.139 & 0.142 & 0.128 & 0.097 & 0.175 & 0.136 \\
\hline Within & 0.035 & 0.026 & 0.030 & 0.037 & 0.037 & 0.029 & 0.020 & 0.059 & 0.047 \\
\hline Between & 0.103 & 0.081 & 0.094 & 0.102 & 0.106 & 0.100 & 0.077 & 0.116 & 0.090 \\
\hline \multicolumn{10}{|c|}{ Environment } \\
\hline Theil & 0.725 & 0.714 & 0.788 & 0.797 & 0.599 & 0.543 & 0.492 & 0.196 & 0.214 \\
\hline Within & 0.540 & 0.615 & 0.661 & 0.675 & 0.508 & 0.480 & 0.461 & 0.183 & 0.200 \\
\hline Between & 0.186 & 0.099 & 0.127 & 0.122 & 0.091 & 0.064 & 0.031 & 0.013 & 0.014 \\
\hline \multicolumn{10}{|c|}{ Essential public services } \\
\hline Theil & 0.162 & 0.180 & 0.216 & 0.157 & 0.158 & 0.094 & 0.114 & 0.114 & 0.172 \\
\hline Within & 0.057 & 0.078 & 0.125 & 0.076 & 0.105 & 0.034 & 0.061 & 0.055 & 0.088 \\
\hline Between & 0.105 & 0.103 & 0.091 & 0.081 & 0.053 & 0.059 & 0.053 & 0.060 & 0.084 \\
\hline \multicolumn{10}{|l|}{ Health } \\
\hline Theil & 0.144 & 0.140 & 0.075 & 0.212 & 0.170 & 0.125 & 0.147 & 0.223 & 0.217 \\
\hline Within & 0.025 & 0.055 & 0.019 & 0.124 & 0.047 & 0.028 & 0.036 & 0.125 & 0.061 \\
\hline Between & 0.118 & 0.084 & 0.056 & 0.088 & 0.124 & 0.097 & 0.112 & 0.098 & 0.156 \\
\hline \multicolumn{10}{|c|}{ Material living conditions } \\
\hline Theil & 0.083 & 0.102 & 0.120 & 0.123 & 0.091 & 0.121 & 0.098 & 0.072 & 0.072 \\
\hline Within & 0.030 & 0.041 & 0.053 & 0.055 & 0.037 & 0.045 & 0.041 & 0.030 & 0.030 \\
\hline Between & 0.053 & 0.062 & 0.067 & 0.068 & 0.054 & 0.075 & 0.057 & 0.042 & 0.042 \\
\hline \multicolumn{10}{|c|}{ Personal security } \\
\hline Theil & 0.029 & 0.027 & 0.038 & 0.045 & 0.088 & 0.064 & 0.134 & 0.209 & 0.065 \\
\hline Within & 0.028 & 0.027 & 0.036 & 0.045 & 0.086 & 0.063 & 0.133 & 0.207 & 0.065 \\
\hline Between & 0.001 & 0.000 & 0.003 & 0.000 & 0.001 & 0.001 & 0.000 & 0.002 & 0.000 \\
\hline
\end{tabular}


Table 7 (continued)

\begin{tabular}{|c|c|c|c|c|c|c|c|c|c|}
\hline & 2004 & 2005 & 2006 & 2007 & 2008 & 2009 & 2010 & 2011 & 2012 \\
\hline \multicolumn{10}{|c|}{ Research and innovation } \\
\hline Theil & 0.233 & 0.256 & 0.269 & 0.262 & 0.248 & 0.246 & 0.241 & 0.336 & 0.279 \\
\hline Within & 0.090 & 0.089 & 0.099 & 0.098 & 0.088 & 0.063 & 0.072 & 0.096 & 0.066 \\
\hline Between & 0.143 & 0.167 & 0.170 & 0.164 & 0.160 & 0.183 & 0.169 & 0.240 & 0.214 \\
\hline \multicolumn{10}{|c|}{ Social relations } \\
\hline Theil & 0.277 & 0.282 & 0.248 & 0.233 & 0.230 & 0.251 & 0.162 & 0.249 & 0.213 \\
\hline Within & 0.111 & 0.124 & 0.095 & 0.098 & 0.085 & 0.101 & 0.070 & 0.095 & 0.064 \\
\hline Between & 0.166 & 0.158 & 0.153 & 0.135 & 0.145 & 0.151 & 0.092 & 0.154 & 0.149 \\
\hline \multicolumn{10}{|l|}{$R W B I$} \\
\hline Theil & 0.213 & 0.195 & 0.156 & 0.165 & 0.173 & 0.169 & 0.167 & 0.224 & 0.199 \\
\hline Within & 0.103 & 0.091 & 0.085 & 0.072 & 0.096 & 0.068 & 0.073 & 0.075 & 0.057 \\
\hline Between & 0.110 & 0.105 & 0.071 & 0.093 & 0.076 & 0.100 & 0.094 & 0.149 & 0.143 \\
\hline \multicolumn{10}{|c|}{ Per-capita GDP } \\
\hline Theil & 0.227 & 0.270 & 0.259 & 0.259 & 0.262 & 0.259 & 0.256 & 0.264 & 0.252 \\
\hline Within & 0.034 & 0.044 & 0.044 & 0.046 & 0.047 & 0.050 & 0.047 & 0.059 & 0.057 \\
\hline Between & 0.193 & 0.226 & 0.215 & 0.213 & 0.215 & 0.209 & 0.208 & 0.205 & 0.195 \\
\hline
\end{tabular}

Table 8 Theil index by subnational areas. Source: Our elaborations on Ferrara and Nisticò (2015) and Istat data

\begin{tabular}{|c|c|c|c|c|c|c|c|c|c|}
\hline & 2004 & 2005 & 2006 & 2007 & 2008 & 2009 & 2010 & 2011 & 2012 \\
\hline \multicolumn{10}{|c|}{ Culture and free-time } \\
\hline North & 0.020 & 0.042 & 0.033 & 0.019 & 0.028 & 0.032 & 0.022 & 0.023 & 0.021 \\
\hline Centre & 0.014 & 0.022 & 0.006 & 0.022 & 0.027 & 0.012 & 0.026 & 0.029 & 0.015 \\
\hline Mezzogiorno & 0.106 & 0.260 & 0.223 & 0.194 & 0.323 & 0.289 & 0.352 & 0.279 & 0.526 \\
\hline \multicolumn{10}{|l|}{ Education } \\
\hline North & 0.191 & 0.184 & 0.051 & 0.095 & 0.110 & 0.040 & 0.140 & 0.035 & 0.159 \\
\hline Centre & 0.025 & 0.012 & 0.022 & 0.058 & 0.023 & 0.007 & 0.059 & 0.012 & 0.007 \\
\hline Mezzogiorno & 0.326 & 0.262 & 0.059 & 0.095 & 0.045 & 0.102 & 0.139 & 0.168 & 0.065 \\
\hline \multicolumn{10}{|l|}{ Employment } \\
\hline North & 0.004 & 0.002 & 0.003 & 0.004 & 0.003 & 0.003 & 0.003 & 0.002 & 0.003 \\
\hline Centre & 0.013 & 0.010 & 0.013 & 0.014 & 0.011 & 0.009 & 0.006 & 0.011 & 0.008 \\
\hline Mezzogiorno & 0.175 & 0.116 & 0.148 & 0.189 & 0.198 & 0.146 & 0.088 & 0.310 & 0.241 \\
\hline \multicolumn{10}{|l|}{ Environment } \\
\hline North & 0.711 & 0.905 & 0.914 & 0.969 & 0.720 & 0.716 & 0.808 & 0.373 & 0.409 \\
\hline Centre & 0.074 & 0.125 & 0.155 & 0.245 & 0.082 & 0.070 & 0.140 & 0.027 & 0.020 \\
\hline Mezzogiorno & 0.247 & 0.153 & 0.207 & 0.110 & 0.214 & 0.202 & 0.080 & 0.048 & 0.058 \\
\hline \multicolumn{10}{|c|}{ Essential public services } \\
\hline North & 0.041 & 0.008 & 0.007 & 0.008 & 0.009 & 0.006 & 0.010 & 0.026 & 0.020 \\
\hline Centre & 0.040 & 0.029 & 0.037 & 0.008 & 0.047 & 0.015 & 0.046 & 0.016 & 0.013 \\
\hline Mezzogiorno & 0.137 & 0.334 & 0.534 & 0.314 & 0.351 & 0.127 & 0.203 & 0.173 & 0.351 \\
\hline
\end{tabular}


Table 8 (continued)

\begin{tabular}{|c|c|c|c|c|c|c|c|c|c|}
\hline & 2004 & 2005 & 2006 & 2007 & 2008 & 2009 & 2010 & 2011 & 2012 \\
\hline \multicolumn{10}{|l|}{ Health } \\
\hline North & 0.005 & 0.008 & 0.003 & 0.050 & 0.026 & 0.006 & 0.013 & 0.029 & 0.006 \\
\hline Centre & 0.008 & 0.013 & 0.010 & 0.017 & 0.010 & 0.013 & 0.032 & 0.033 & 0.006 \\
\hline Mezzogiorno & 0.132 & 0.262 & 0.065 & 0.479 & 0.191 & 0.125 & 0.127 & 0.519 & 0.381 \\
\hline \multicolumn{10}{|c|}{ Material living conditions } \\
\hline North & 0.001 & 0.003 & 0.002 & 0.002 & 0.001 & 0.001 & 0.001 & 0.002 & 0.002 \\
\hline Centre & 0.004 & 0.005 & 0.006 & 0.004 & 0.003 & 0.004 & 0.001 & 0.003 & 0.003 \\
\hline Mezzogiorno & 0.131 & 0.183 & 0.250 & 0.266 & 0.165 & 0.229 & 0.187 & 0.120 & 0.120 \\
\hline \multicolumn{10}{|c|}{ Personal security } \\
\hline North & 0.020 & 0.022 & 0.045 & 0.046 & 0.051 & 0.050 & 0.116 & 0.193 & 0.031 \\
\hline Centre & 0.017 & 0.018 & 0.036 & 0.036 & 0.025 & 0.027 & 0.071 & 0.106 & 0.034 \\
\hline Mezzogiorno & 0.043 & 0.038 & 0.026 & 0.048 & 0.170 & 0.099 & 0.189 & 0.265 & 0.123 \\
\hline \multicolumn{10}{|c|}{ Research and innovation } \\
\hline North & 0.095 & 0.085 & 0.093 & 0.101 & 0.088 & 0.047 & 0.065 & 0.076 & 0.068 \\
\hline Centre & 0.014 & 0.016 & 0.015 & 0.011 & 0.021 & 0.034 & 0.030 & 0.028 & 0.027 \\
\hline Mezzogiorno & 0.182 & 0.251 & 0.285 & 0.249 & 0.201 & 0.212 & 0.183 & 0.375 & 0.132 \\
\hline \multicolumn{10}{|c|}{ Social relations } \\
\hline North & 0.088 & 0.106 & 0.068 & 0.050 & 0.053 & 0.067 & 0.049 & 0.046 & 0.042 \\
\hline Centre & 0.051 & 0.047 & 0.046 & 0.106 & 0.036 & 0.053 & 0.044 & 0.045 & 0.038 \\
\hline Mezzogiorno & 0.315 & 0.333 & 0.295 & 0.294 & 0.289 & 0.345 & 0.165 & 0.349 & 0.188 \\
\hline \multicolumn{10}{|l|}{$R W B I$} \\
\hline North & 0.085 & 0.050 & 0.082 & 0.026 & 0.058 & 0.036 & 0.024 & 0.057 & 0.029 \\
\hline Centre & 0.019 & 0.017 & 0.018 & 0.014 & 0.014 & 0.012 & 0.015 & 0.003 & 0.006 \\
\hline Mezzogiorno & 0.271 & 0.331 & 0.157 & 0.298 & 0.292 & 0.248 & 0.312 & 0.265 & 0.242 \\
\hline \multicolumn{10}{|c|}{ Per-capita GDP } \\
\hline North & 0.011 & 0.011 & 0.011 & 0.010 & 0.012 & 0.016 & 0.016 & 0.021 & 0.025 \\
\hline Centre & 0.023 & 0.025 & 0.019 & 0.018 & 0.018 & 0.028 & 0.023 & 0.041 & 0.039 \\
\hline Mezzogiorno & 0.263 & 0.373 & 0.365 & 0.392 & 0.383 & 0.358 & 0.329 & 0.368 & 0.301 \\
\hline
\end{tabular}

\section{References}

Acemoglu, D., Johnson, S., \& Robinson, J. A. (2001). The colonial origins of comparative development: An empirical investigation. American Economic Review, 91, 1369-1401.

Acemoglu, D., Johnson, S., \& Robinson, J. A. (2005). Institutions as a fundamental cause of long-run growth. In P. Aghion \& S. Durlauf (Eds.), Handbook of economic growth (pp. 385-472). Amsterdam: Elsevier.

Aghion, P., \& Durlauf, S. (Eds.). (2005). Handbook of economic growth. Amsterdam: Elsevier.

Ahn, T. K., \& Ostrom, E. (2009). Social capital and collective action. In D. Castiglione, J. W. Van Deth, \& G. Wolleb (Eds.), The handbook of social capital. Oxford: Oxford University Press.

Aimone Gigio, L., Alampi, D., Camussi, S., Ciaccio, G., Guaitini, P., Lozzi, M., Mancini, A. L., Panicara, E. \& Paolicelli, M. (2018). La sanità in Italia: il difficile equilibrio tra vincoli di bilancio e qualità dei servizi nelle regioni in piano di rientro. In Questioni di Economia e Finanza (Occasional Papers), Vol. 427, Rome, Bank of Italy.

Amin, A., \& Thrift, N. (1995). Globalization, institutional thickness and the local economy. In P. Healy, S. Cameron, \& A. Davoudi (Eds.), Managing cities: The new urban context (pp. 92-108). Chichester: Wiley. 
Anselin, L., Le Gallo, J., \& Jayet, H. (2008). Spatial panel econometrics. In The econometrics of panel data (pp. 625-660). Berlin: Springer.

Aoki, M. (2001). Toward a comparative institutional analysis. Cambridge, MA: MIT Press.

Barro, R. (1997). Determinants of economic growth. Cambridge, MA: MIT Press.

Belotti, F., Hughes, G., \& Piano Mortari, A. (2017). XSMLE: Stata module for spatial panel data models estimation.https://EconPapers.repec.org/RePEc:boc:bocode:s457610. Accessed 15 Apr 2019.

Berloffa, G., \& Modena, F. (2012). Economic well-being in Italy: The role of income insecurity and intergenerational inequality. Journal of Economic Behavior \& Organization, 81(3), 751-765.

Bourguignon, F., \& Morrisson, C. (2002). Inequality among world citizens: 1820-1992. American Economic Review, 92(4), 727-744.

Brandolini, A. \& Vecchi, G. (2011). The well-being of Italians: A comparative historical approach. In Economic history working papers, 19, Rome, Bank of Italy.

Cafagna, L. (1988). Modernizzazione attiva e modernizzazione passiva. Meridiana, 2, 229-240.

Cannari, L., \& Franco, D. (2011). Il Mezzogiorno: ritardi, qualità dei servizi pubblici, politiche. Stato $e$ mercato, 1, 3-40.

Capriati, M. (2011). Spesa pubblica e sviluppo umano nelle regioni italiane. QA La Questione Agraria, 2, 23-56.

Cersosimo, D., \& Nisticò, R. (2013). Un Paese disuguale: il divario civile in Italia. Stato e mercato, 33(2), 265-300.

Charron, N., Dijkstra, L., \& Lapuente, V. (2014). Regional governance matters: Quality of government within European Union member states. Regional Studies, 48(1), 68-90.

Corsi, M., \& Guarini, G. (2011). Measuring progress of Italian regions: A classical approach. Ekonomiaz, 78(03), 340-367.

Cortinovis, N., Xiao, J., Boschma, R., \& van Oort, F. G. (2017). Quality of government and social capital as drivers of regional diversification in Europe. Journal of Economic Geography, 17(6), 1179-1208.

Cowell, F. (2011). Measuring inequality. Oxford University Press.

Crescenzi, R., Di Cataldo, M., \& Rodríguez-Pose, A. (2016). Government quality and the economic returns of transport infrastructure investment in European regions. Journal of Regional Science, 56(4), 555-582.

Decancq, K., Decoster, A., \& Schokkaert, E. (2009). The evolution of world inequality in well-being. World Development, 37(1), 11-25.

Dei Ottati, G. (1994). Cooperation and competition in the industrial districts as an organization model. European Planning Studies, 2, 463-483.

Dellepiane-Avellaneda, S. (2010). Good governance, institutions and economic development: Beyond the conventional wisdom. British Journal of Political Science, 40(1), 195-224.

Di Berardino, C., Mauro, G., Quaglione, D., \& Sarra, A. (2016). Industrial districts and socio-economic well-being: An investigation on the Italian provinces disparities. Social Indicators Research, 129(1), 337-363.

Dialga, I. (2017). Highlighting methodological limitations in the steps of composite indicators construction. Social Indicators Research, 131(2), 441-465.

Elhorst, J. P. (2014). Spatial panel data models. In Spatial econometrics (pp. 37-93). Berlin: Springer.

European Commission (2014). Investment for jobs and growth. In Promoting development and good governance in EU regions and cities, sixth report on economic, social and territorial cohesion. Luxembourg: Publications Office of the European Union.

European Commission (2017). My region, my Europe, our future. In Seventh report on economic, social and territorial cohesion. Luxembourg: Publications Office of the European Union.

Ezcurra, R., \& Rodrìguez-Pose, A. (2014). Government quality and spatial inequality: A cross-country analysis. Environment Planning, 46, 1732-1753.

Felice, E. (2007). I divari regionali in Italia sulla base degli indicatori sociali (1871-2001). Rivista di politica economica, 97(3/4), 359.

Felice, E. (2017). Il divario socio-istituzionale tra Nord e Sud. Un'interpretazione di lungo periodo. In M. Marini (Ed.), Le politiche di coesione territoriale. Un confronto tra Italia e Stati Uniti d'America. Soveria Mannelli: Rubbettino.

Felice, E., \& Vasta, M. (2015). Passive modernization? The New Human Development Index and its components in Italy's regions (1871-2007). European Review of Economic History, 19(1), 44-66.

Ferrara, A. R., \& Nisticò, R. (2013). Well-being indicators and convergence across Italian regions. Applied Research in Quality of Life, 8, 15-44.

Ferrara, A. R., \& Nisticò, R. (2015). Regional well-being indicators and dispersion from a multidimensional perspective: Evidence from Italy. The Annals of Regional Science, 55(2), 373-420. 
Francese, M., \& Romanelli, M. (2011). Healthcare in Italy: Expenditure determinants and Regional Differentials. In Temi di Discussione (Working Papers), 828, Rome, Bank of Italy.

Frey, B. S., \& Stutzer, A. (2000). Happiness, economy and institutions. Economic Journal, 110, 918-938.

Golden, M. A., \& Picci, L. (2005). Proposal for a new measure of corruption, illustrated with Italian data. Economics and Politics, 17, 37-75.

Greif, A. (1989). Reputation and coalitions in medieval trade: Evidence on the Maghribi traders. Journal of Economic History, 49, 857-882.

Greif, A. (1998). Historical and comparative institutional analysis. The American Economic Review, 88(2), $80-84$.

Greif, A., Milgrom, R., \& Weingast, B. (1994). Coordination, commitment and enforcement: The case of merchant guild. Journal of Political Economy, 102, 745-776.

Gupta, S., Davoodi H. R., \& Alonso-Terme, R. (1998). Does corruption affect income inequality and poverty? In Working paper number 98/76. Washington, DC: International Monetary Fund (IMF).

Hagerty, M. R., \& Land, K. C. (2007). Constructing summary indices of quality of life: A model for the effect of heterogeneous importance weights. Sociological Methods and Research, 35(4), 455-496.

Hall, R. E., \& Jones, C. I. (1999). Why do some countries produce so much more output per worker than others? Quarterly Journal of Economics, 114, 83-116.

Hall, P. A., \& Taylor, R. C. (1996). Political science and the three new institutionalisms. Political Studies, 44(5), 936-957.

Harrison, L., \& Huntington, S. (Eds.). (2000). Culture matters: How values shape human progress. New York: Basic Books.

Helliwell, J. F., \& Huang, H. (2008). How's your government? International evidence linking good government and well-being. British Journal of Political Science, 38, 595-619.

Hodgson, G. (2007). The revival of Veblenian institutional economics. Journal of Economic Issues, 2, $325-340$.

Holmberg, S., Rothstein, B., \& Nasiritousi, N. (2009). Quality of government: What you get. Annual Review of Political Science, 12, 135-161.

Hurwicz, L. (1996). Institutions as families of game forms. The Japanese Economic Review, 47(2), $113-132$.

Iammarino, S., Rodriguez-Pose, A., \& Storper, M. (2018). Regional inequality in Europe: Evidence, theory and policy implications. Journal of Economic Geography, 19(2), 273-298.

Iuzzolino, G., Pellegrini, G., \& Viesti, G. (2011). Convergence among Italian regions, 1861-2011. In Economic history working papers 22. Rome: Bank of Italy.

Kaufmann, D., Kraay, A., \& Mastruzzi, M. (2011). The worldwide governance indicators: Methodology and analytical issues. Hague Journal on the Rule of Law, 3(2), 220-246.

Kunčič, A. (2014). Institutional quality dataset. Journal of Institutional Economics, 10(1), 135-161.

Kyriacou, A., Muinelo-Gallo, L., \& Roca-Sagalés, O. (2015). Fiscal decentralization and regional disparities: The importance of good governance. Papers in Regional Science, 94, 89-107.

Kyriacou, A., \& Roca-Sagalés, O. (2013). Regional disparities and government quality: Redistributive conflict crowds out good government. Spatial Economic Analysis, 9, 183-201.

Landes, D. (2000). Culture makes almost all the difference. In L. Harrison \& S. Huntington (Eds.), Culture matters: How values shape human progress (pp. 2-13). New York: Basic Books.

LeSage, J., \& Pace, R. K. (2009). Introduction to spatial econometrics. Boca Raton: Chapman and Hall/ CRC.

Lucas, R. E. (1988). On the mechanics of economic development. Journal of Monetary Economics, 22, 3-42.

Marchante, A. J., \& Ortega, B. (2006). Quality of life and economic convergence across Spanish regions, 1980-2001. Regional Studies, 40(5), 471-483.

Marchante, A. J., Ortega, B., \& Sánchez, J. (2006). The evolution of well-being in Spain (1980-2001): A regional analysis. Social Indicators Research, 76(2), 283-316.

Mauro, P. (1995). Corruption and growth. The Quarterly Journal of Economics, 110(3), 681-712.

Mauro, P. (1998). Corruption and composition of government expenditure. Journal of Public Economics, 69, 263-279.

Mo, P. H. (2001). Corruption and economic growth. Journal of Public Economics, 29, 66-79.

Moran, P. A. (1950). Notes on continuous stochastic phenomena. Biometrika, 37(1/2), 17-23.

Morse, S. (2006). Is corruption bad for environmental sustainability? A cross-national analysis. Ecology and Society, 11(1), 22.

Mundlak, Y. (1978). On the pooling of time series and cross section data. Econometrica: Journal of the Econometric Society, 1, 69-85. 
Nardo, M., Saisana, M., Saltelli, A., Tarantola, S., Hoffman, A., Giovannini, E. (2008). Handbook on constructing composite indicators: Methodology and user guide. OECD publishing.

Nelson, R. (1994). The co-evolution of technology, industrial structure, and supporting institutions. Industrial and Corporate Change, 3, 47-63.

Nicoletti, G., Scarpetta, S., \& Boylaud, O. (2000). Summary indicators of product market regulation with an extension to employment protection legislation. In OECD Economics department working papers, no. 226. Paris: OECD Publishing.

Nifo, A., \& Vecchione, G. (2014). Do institutions play a role in skilled migration? The Case of Italy, Regional Studies, 48(10), 1628-1649.

North, D. C. (1990). Institutions, institutional change and economic performance. New York: Cambridge University Press.

North, D. C. (1991). Institutions. Journal of economic perspectives, 5(1), 97-112.

OECD. (2016). PISA 2015 results (Volume I): Excellence and equity in education. Paris: OECD Publishing.

Ostrom, E. (2015). Governing the commons. Cambridge: Cambridge University Press.

Ott, J. C. (2010). Good governance and happiness in nations: Technical quality precedes democracy and quality beats size. Journal of Happiness Studies, 11, 353-368.

Putnam, R. D. (1993). Making democracy work: Civic traditions in modern Italy. Princeton, NJ: Princeton University Press.

Robinson, J. A., \& Acemoglu, D. (2012). Why nations fail: The origins of power, prosperity and poverty. New York: Crown Business.

Rodríguez-Pose, A. (2013). Do institutions matter for regional development? Regional Studies, 47(7), 1034-1047.

Rodríguez-Pose, A., \& Di Cataldo, M. (2015). Quality of government and innovative performance in the regions of Europe. Journal of Economic Geography, 15, 673-706.

Rodríguez-Pose, A., \& Garcilazo, E. (2015). Quality of government and the returns of investment: Examining the impact of cohesion expenditure in European regions. Regional Studies, 49(8), 1274-1290.

Rodríguez-Pose, A., \& Maslauskaite, K. (2012). Can policy make us happier? Individual characteristics, socioeconomic factors, and life satisfaction in Central and Eastern Europe. Cambridge Journal of Regions Economy and Society, 5, 77-96.

Rodríguez-Pose, A., \& Storper, M. (2006). Better rules or stronger communities? On the social foundations of institutional change and its economic effects. Economic Geography, 82, 1-25.

Rodríguez-Pose, A., \& Tselios, V. (2015). Toward inclusive growth: Is there regional convergence in social welfare? International Regional Science Review, 38(1), 30-60.

Rodrik, D., Subramanian, F., \& Trebbi, F. (2004). Institutions rule: The primacy of institutions over geography and integration in economic development. Journal of Economic Growth, 9, 131-165.

Romer, P. M. (1986). Increasing returns and long-run growth. Journal of Political Economy, 94, $1002-1037$.

Saisana, M., Saltelli, A., \& Tarantola, S. (2005). Uncertainty and sensitivity analysis techniques as tools for the quality assessment of composite indicators. Journal of the Royal Statistical Society: Series A (Statistics in Society), 168(2), 307-323.

Solow, R. M. (1956). A contribution to the theory of economic growth. Quarterly Journal of Economics, 70, 65-94.

Stiglitz, J., Sen, A., \& Fitoussi, J. P. (2009). Report by the commission on the measurement of economic performance and social progress. Paris

Storper, M. (1997). The regional world: Territorial development in a global economy. New York, NY: Guildford.

Trigilia, C. (1990). Work and politics in the third Italy's industrial districts. In F. Pyke, G. Becattini, \& W. Sengenberger (Eds.), Industrial districts and inter-firm co-operation in Italy (pp. 160-184). Geneva: International Institute for Labour Studies (ILO).

Trigilia, C. (2015). Cultura, istituzioni e sviluppo. La lezione di Max Weber e il neo-istituzionalismo. Stato e mercato, 104, 263-280.

Vijayaraghavan, M., \& Ward, W. A. (2001). Institutions and economic growth: Empirical evidence from a cross-national analysis. In Working paper number 001302. Clemson, SC: Center for International Trade, Clemson University.

Welsch, H. (2004). Corruption, growth, and the environment: A cross-country analysis. Environmental Development Economics, 9, 663-693. 
Williamson, O. E. (1989). Transaction cost economics. Handbook of Industrial Organization, 1, $135-182$.

Wooldridge, J. M. (2010). Econometric analysis of cross section and panel data. Cambridge: MIT Press.

Publisher's Note Springer Nature remains neutral with regard to jurisdictional claims in published maps and institutional affiliations. 